\title{
Composite Iterative Algorithms for Variational Inequality and Fixed Point Problems in Real Smooth and Uniformly Convex Banach Spaces
}

\author{
Lu-Chuan Ceng ${ }^{1}$ and Ching-Feng Wen ${ }^{2}$ \\ ${ }^{1}$ Department of Mathematics, Shanghai Normal University and Scientific Computing Key Laboratory of Shanghai Universities, \\ Shanghai 200234, China \\ ${ }^{2}$ Center for Fundamental Science, Kaohsiung Medical University, Kaohsiung 807, Taiwan
}

Correspondence should be addressed to Ching-Feng Wen; cfwen@kmu.edu.tw

Received 30 April 2013; Accepted 6 June 2013

Academic Editor: Wei-Shih Du

Copyright (C) 2013 L.-C. Ceng and C.-F. Wen. This is an open access article distributed under the Creative Commons Attribution License, which permits unrestricted use, distribution, and reproduction in any medium, provided the original work is properly cited.

We introduce composite implicit and explicit iterative algorithms for solving a general system of variational inequalities and a common fixed point problem of an infinite family of nonexpansive mappings in a real smooth and uniformly convex Banach space. These composite iterative algorithms are based on Korpelevich's extragradient method and viscosity approximation method. We first consider and analyze a composite implicit iterative algorithm in the setting of uniformly convex and 2-uniformly smooth Banach space and then another composite explicit iterative algorithm in a uniformly convex Banach space with a uniformly Gâteaux differentiable norm. Under suitable assumptions, we derive some strong convergence theorems. The results presented in this paper improve, extend, supplement, and develop the corresponding results announced in the earlier and very recent literatures.

\section{Introduction}

Let $X$ be a real Banach space whose dual space is denoted by $X^{*}$. The normalized duality mapping $J: X \rightarrow 2^{X^{*}}$ is defined by

$$
J(x)=\left\{x^{*} \in X^{*}:\left\langle x, x^{*}\right\rangle=\|x\|^{2}=\left\|x^{*}\right\|^{2}\right\}, \quad \forall x \in X,
$$

where $\langle\cdot, \cdot\rangle$ denotes the generalized duality pairing. It is an immediate consequence of the Hahn-Banach theorem that $J(x)$ is nonempty for each $x \in X$. Let $C$ be a nonempty, closed, and convex subset of $X$. A mapping $T: C \rightarrow C$ is called nonexpansive if $\|T x-T y\| \leq\|x-y\|$ for every $x, y \in C$. The set of fixed points of $T$ is denoted by $\operatorname{Fix}(T)$. We use the notation $\rightarrow$ to indicate the weak convergence and the one $\rightarrow$ to indicate the strong convergence. A mapping $A: C \rightarrow X$ is said to be accretive if for each $x, y \in C$, there exists $j(x-y) \in$ $J(x-y)$ such that

$$
\langle A x-A y, j(x-y)\rangle \geq 0 .
$$

It is said to be $\alpha$-strongly accretive if for each $x, y \in C$, there exists $j(x-y) \in J(x-y)$ such that

$$
\langle A x-A y, j(x-y)\rangle \geq \alpha\|x-y\|^{2},
$$

for some $\alpha \in(0,1)$. The mapping is called $\beta$-inverse stronglyaccretive if for each $x, y \in C$, there exists $j(x-y) \in J(x-y)$ such that

$$
\langle A x-A y, j(x-y)\rangle \geq \beta\|A x-A y\|^{2},
$$

for some $\beta>0$ and is said to be $\lambda$-strictly pseudocontractive if for each $x, y \in C$, there exists $j(x-y) \in J(x-y)$ such that

$$
\langle A x-A y, j(x-y)\rangle \leq\|x-y\|^{2}-\lambda\|x-y-(A x-A y)\|^{2}
$$

for some $\lambda \in(0,1)$. 
Let $U=\{x \in X:\|x\|=1\}$ denote the unite sphere of $X$. A Banach space $X$ is said to be uniformly convex if for each $\epsilon \in(0,2]$, there exists $\delta>0$ such that for all $x, y \in U$,

$$
\|x-y\| \geq \epsilon \Longrightarrow \frac{\|x+y\|}{2} \leq 1-\delta .
$$

It is known that a uniformly convex Banach space is reflexive and strict convex. A Banach space $X$ is said to be smooth if the limit

$$
\lim _{t \rightarrow 0} \frac{\|x+t y\|-\|x\|}{t}
$$

exists for all $x, y \in U$; in this case, $X$ is also said to have a Gâteaux differentiable norm. $X$ is said to have a uniformly Gâteaux differentiable norm if for each $y \in U$, the limit is attained uniformly for $x \in U$. Moreover, it is said to be uniformly smooth if this limit is attained uniformly for $x, y \in$ $U$. The norm of $X$ is said to be the Fréchet differential if for each $x \in U$, this limit is attained uniformly for $y \in U$. In addition, we define a function $\rho:[0, \infty) \rightarrow[0, \infty)$ called the modulus of smoothness of $X$ as follows:

$$
\begin{aligned}
& \rho(\tau)=\sup \left\{\frac{1}{2}(\|x+y\|+\|x-y\|)\right. \\
&-1: x, y \in X,\|x\|=1,\|y\|=\tau\} .
\end{aligned}
$$

It is known that $X$ is uniformly smooth if and only if $\lim _{\tau \rightarrow 0} \rho(\tau) / \tau=0$. Let $q$ be a fixed real number with $1<q \leq$ 2 . Then a Banach space $X$ is said to be $q$-uniformly smooth if there exists a constant $c>0$ such that $\rho(\tau) \leq c \tau^{q}$ for all $\tau>0$. As pointed out in [1], no Banach space is $q$-uniformly smooth for $q>2$. In addition, it is also known that $J$ is single-valued if and only if $X$ is smooth, whereas if $X$ is uniformly smooth, then the mapping $J$ is norm-to-norm uniformly continuous on bounded subsets of $X$. If $X$ has a uniformly Gâteaux differentiable norm, then the duality mapping $J$ is norm-toweak $^{*}$ uniformly continuous on bounded subsets of $X$.

Very recently, Cai and $\mathrm{Bu}$ [2] considered the following general system of variational inequalities (GSVI) in a real smooth Banach space $X$, which involves finding $\left(x^{*}, y^{*}\right) \in$ $C \times C$ such that

$$
\begin{aligned}
& \left\langle\mu_{1} B_{1} y^{*}+x^{*}-y^{*}, J\left(x-x^{*}\right)\right\rangle \geq 0, \quad \forall x \in C, \\
& \left\langle\mu_{2} B_{2} x^{*}+y^{*}-x^{*}, J\left(x-y^{*}\right)\right\rangle \geq 0, \quad \forall x \in C,
\end{aligned}
$$

where $C$ is a nonempty, closed, and convex subset of $X, B_{1}$, and $B_{2}: C \rightarrow X$ are two nonlinear mappings, and $\mu_{1}$ and $\mu_{2}$ are two positive constants. Here the set of solutions of GSVI (9) is denoted by $\operatorname{GSVI}\left(C, B_{1}, B_{2}\right)$. In particular, if $X=H$, a real Hilbert space, then GSVI (9) reduces to the following GSVI of finding $\left(x^{*}, y^{*}\right) \in C \times C$ such that

$$
\begin{array}{ll}
\left\langle\mu_{1} B_{1} y^{*}+x^{*}-y^{*}, x-x^{*}\right\rangle \geq 0, & \forall x \in C, \\
\left\langle\mu_{2} B_{2} x^{*}+y^{*}-x^{*}, x-y^{*}\right\rangle \geq 0, & \forall x \in C,
\end{array}
$$

which $\mu_{1}$ and $\mu_{2}$ are two positive constants. The set of solutions of problem (10) is still denoted by $\operatorname{GSVI}\left(C, B_{1}, B_{2}\right)$.
It is clear that the problem (10) covers as special case the classical variational inequality problem (VIP) of finding $x^{*} \epsilon$ $C$ such that

$$
\left\langle A x^{*}, x-x^{*}\right\rangle \geq 0, \quad \forall x \in C .
$$

The solution set of the VIP (11) is denoted by $\operatorname{VI}(C, A)$.

Recently, Ceng et al. [3] transformed problem (10) into a fixed point problem in the following way.

Lemma 1 (see [3]). For given $\bar{x}, \bar{y} \in C,(\bar{x}, \bar{y})$ is a solution of problem (10) if and only if $\bar{x}$ is a fixed point of the mapping $G: C \rightarrow C$ defined by

$$
\begin{aligned}
G(x)=P_{C}[ & P_{C}\left(x-\mu_{2} B_{2} x\right)-\mu_{1} B_{1} P_{C} \\
& \left.\times\left(x-\mu_{2} B_{2} x\right)\right], \quad \forall x \in C,
\end{aligned}
$$

where $\bar{y}=P_{C}\left(\bar{x}-\mu_{2} B_{2} \bar{x}\right)$ and $P_{C}$ is the the projection of $H$ onto C.

In particular, if the mappings $B_{i}: C \rightarrow H$ is $\beta_{i}$-inverse strongly monotone for $i=1,2$, then the mapping $G$ is nonexpansive provided $\mu_{i} \in\left(0,2 \beta_{i}\right)$ for $i=1,2$.

Let $C$ be a nonempty, closed, and convex subset of a real smooth Banach space $X$. Let $\Pi_{C}$ be a sunny nonexpansive retraction from $X$ onto $C$, and let $f: C \rightarrow C$ be a contraction with coefficient $\rho \in(0,1)$. In this paper we introduce composite implicit and explicit iterative algorithms for solving GSVI (9) and the common fixed point problem of an infinite family $\left\{S_{n}\right\}$ of nonexpansive mappings of $C$ into itself. These composite iterative algorithms are based on Korpelevich's extragradient method [4] and viscosity approximation method [5]. Let the mapping $G$ be defined by

$$
G(x):=\Pi_{C}\left(I-\mu_{1} B_{1}\right) \Pi_{C}\left(I-\mu_{2} B_{2}\right) x, \quad \forall x \in C .
$$

We first propose a composite implicit iterative algorithm in the setting of uniformly convex and 2-uniformly smooth Banach space $X$ :

$$
\begin{gathered}
y_{n}=\alpha_{n} f\left(y_{n}\right)+\left(1-\alpha_{n}\right) S_{n} G\left(x_{n}\right), \\
x_{n+1}=\beta_{n} x_{n}+\gamma_{n} y_{n}+\delta_{n} S_{n} G\left(y_{n}\right), \quad \forall n \geq 0,
\end{gathered}
$$

where $B_{i}: C \rightarrow X$ is $\alpha_{i}$-inverse-strongly accretive with $0<\mu_{i}<\alpha_{i} / \kappa^{2}$ for $i=1,2$ and $\left\{\alpha_{n}\right\},\left\{\beta_{n}\right\},\left\{\gamma_{n}\right\}$, and $\left\{\delta_{n}\right\}$ are the sequences in $(0,1)$ such that $\beta_{n}+\gamma_{n}+\delta_{n}=1$ for all $n \geq 0$. It is proven that under appropriate conditions, $\left\{x_{n}\right\}$ converges strongly to $q \in F=\bigcap_{i=0}^{\infty} \operatorname{Fix}\left(S_{i}\right) \cap \Omega$, which solves the following VIP:

$$
\langle q-f(q), J(q-p)\rangle \leq 0, \quad \forall p \in F .
$$

On the other hand, we also propose another composite explicit iterative algorithm in a uniformly convex Banach space $X$ with a uniformly Gateaux differentiable norm:

$$
\begin{gathered}
y_{n}=\alpha_{n} G\left(x_{n}\right)+\left(1-\alpha_{n}\right) S_{n} G\left(x_{n}\right), \\
x_{n+1}=\beta_{n} f\left(x_{n}\right)+\gamma_{n} y_{n}+\delta_{n} S_{n} G\left(y_{n}\right), \quad \forall n \geq 0,
\end{gathered}
$$


where $B_{i}: C \rightarrow X$ is $\lambda_{i}$-strictly pseudocontractive and $\alpha_{i}$ strongly accretive with $\alpha_{i}+\lambda_{i} \geq 1$ for $i=1,2$ and $\left\{\alpha_{n}\right\},\left\{\beta_{n}\right\}$, $\left\{\gamma_{n}\right\}$, and $\left\{\delta_{n}\right\}$ are the sequences in $(0,1)$ such that $\beta_{n}+\gamma_{n}+$ $\delta_{n}=1$ for all $n \geq 0$. It is proven that under mild conditions, $\left\{x_{n}\right\}$ also converges strongly to $q \in F=\bigcap_{i=0}^{\infty} \operatorname{Fix}\left(S_{i}\right) \cap \Omega$, which solves the VIP (15). The results presented in this paper improve, extend, supplement, and develop the corresponding results announced in the earlier and very recent literatures.

\section{Preliminaries}

We list some lemmas that will be used in the sequel. Lemma 2 can be found in [6]. Lemma 3 is an immediate consequence of the subdifferential inequality of the function $(1 / 2)\|\cdot\|^{2}$.

Lemma 2. Let $\left\{s_{n}\right\}$ be a sequence of nonnegative real numbers satisfying

$$
s_{n+1} \leq\left(1-\alpha_{n}\right) s_{n}+\alpha_{n} \beta_{n}+\gamma_{n}, \quad \forall n \geq 0,
$$

where $\left\{\alpha_{n}\right\},\left\{\beta_{n}\right\}$, and $\left\{\gamma_{n}\right\}$ satisfy the conditions:

(i) $\left\{\alpha_{n}\right\} \subset[0,1]$ and $\sum_{n=0}^{\infty} \alpha_{n}=\infty$,

(ii) $\lim \sup _{n \rightarrow \infty} \beta_{n} \leq 0$,

(iii) $\gamma_{n} \geq 0, \forall n \geq 0$, and $\sum_{n=0}^{\infty} \gamma_{n}<\infty$.

Then $\lim \sup _{n \rightarrow \infty} s_{n}=0$.

Lemma 3. In a smooth Banach space $X$, there holds the inequality

$$
\|x+y\|^{2} \leq\|x\|^{2}+2\langle y, J(x+y)\rangle, \quad \forall x, y \in X .
$$

Lemma 4 (see [7]). Let $\left\{x_{n}\right\}$ and $\left\{z_{n}\right\}$ be bounded sequences in a Banach space $X$, and let $\left\{\alpha_{n}\right\}$ be a sequence in $[0,1]$ which satisfies the following condition:

$$
0<\liminf _{n \rightarrow \infty} \alpha_{n} \leq \limsup _{n \rightarrow \infty} \alpha_{n}<1 .
$$

Suppose that $x_{n+1}=\alpha_{n} x_{n}+\left(1-\alpha_{n}\right) z_{n}, \forall n \geq 0$, and $\lim \sup _{n \rightarrow \infty}\left(\left\|z_{n+1}-z_{n}\right\|-\left\|x_{n+1}-x_{n}\right\|\right) \leq 0$. Then $\lim _{n \rightarrow \infty}\left\|z_{n}-x_{n}\right\|=0$.

Let $D$ be a subset of $C$, and let $\Pi$ be a mapping of $C$ into $D$. Then $\Pi$ is said to be sunny if

$$
\Pi[\Pi(x)+t(x-\Pi(x))]=\Pi(x),
$$

whenever $\Pi(x)+t(x-\Pi(x)) \in C$ for $x \in C$ and $t \geq 0$. A mapping $\Pi$ of $C$ into itself is called a retraction if $\Pi^{2}=\Pi$. If a mapping $\Pi$ of $C$ into itself is a retraction, then $\Pi(z)=z$ for every $z \in R(\Pi)$ where $R(\Pi)$ is the range of $\Pi$. A subset $D$ of $C$ is called a sunny nonexpansive retract of $C$ if there exists a sunny nonexpansive retraction from $C$ onto $D$. The following lemma concerns the sunny nonexpansive retraction.

Lemma 5 (see [8]). Let $C$ be a nonempty, closed, and convex subset of a real smooth Banach space $X$. Let $D$ be a nonempty subset of $C$. Let $\Pi$ be a retraction of $C$ onto $D$. Then the following are equivalent: (i) $\Pi$ is sunny and nonexpansive;

(ii) $\|\Pi(x)-\Pi(y)\|^{2} \leq\langle x-y, J(\Pi(x)-\Pi(y))\rangle, \forall x, y \in C$;

(iii) $\langle x-\Pi(x), J(y-\Pi(x))\rangle \leq 0, \forall x \in C, y \in D$.

It is well known that if $X=H$ a Hilbert space, then a sunny nonexpansive retraction $\Pi_{C}$ is coincident with the metric projection from $X$ onto $C$; that is, $\Pi_{C}=P_{C}$. If $C$ is a nonempty, closed, and convex subset of a strictly convex and uniformly smooth Banach space $X$ and if $T: C \rightarrow C$ is a nonexpansive mapping with the fixed point set $\operatorname{Fix}(T) \neq \emptyset$, then the set $\operatorname{Fix}(T)$ is a sunny nonexpansive retract of $C$.

Lemma 6 (see [9]). Given a number $r>0$. A real Banach space $X$ is uniformly convex if and only if there exists a continuous strictly increasing function $g:[0, \infty) \rightarrow[0, \infty)$, $g(0)=0$, such that

$$
\begin{aligned}
\| \lambda x+ & (1-\lambda) y \|^{2} \\
& \leq \lambda\|x\|^{2}+(1-\lambda)\|y\|^{2}-\lambda(1-\lambda) g(\|x-y\|)
\end{aligned}
$$

for all $\lambda \in[0,1]$ and $x, y \in X$ such that $\|x\| \leq r$ and $\|y\| \leq r$.

Lemma 7 (see [10]). Let $C$ be a nonempty, closed, and convex subset of a Banach space $X$. Let $S_{0}, S_{1}, \ldots$, be a sequence of mappings ofC into itself. Suppose that $\sum_{n=1}^{\infty} \sup \left\{\left\|S_{n} x-S_{n-1} x\right\|\right.$ : $x \in C\}<\infty$. Then for each $y \in C,\left\{S_{n} y\right\}$ converges strongly to some point of $C$. Moreover, let $S$ be a mapping of $C$ into itself defined by $S y=\lim _{n \rightarrow \infty} S_{n} y$ for all $y \in C$. Then $\lim _{n \rightarrow \infty} \sup \left\{\left\|S x-S_{n} x\right\|: x \in C\right\}=0$.

Let $C$ be a nonempty, closed, and convex subset of a Banach space $X$, and let $T: C \rightarrow C$ be a nonexpansive mapping with $\operatorname{Fix}(T) \neq \emptyset$. As previous, let $\Xi_{C}$ be the set of all contractions on $C$. For $t \in(0,1)$ and $f \in \Xi_{C}$, let $x_{t} \in C$ be the unique fixed point of the contraction $x \mapsto t f(x)+(1-t) T x$ on $C$; that is,

$$
x_{t}=t f\left(x_{t}\right)+(1-t) T x_{t} .
$$

Lemma 8 (see $[11,12])$. Let $X$ be a uniformly smooth Banach space or a reflexive and strictly convex Banach space with a uniformly Gateaux differentiable norm. Let $C$ be a nonempty, closed, and convex subset of $X$, let $T: C \rightarrow C$ be a nonexpansive mapping with $\operatorname{Fix}(T) \neq \emptyset$, and $f \in \Xi_{C}$. Then the net $\left\{x_{t}\right\}$ defined by $x_{t}=t f\left(x_{t}\right)+(1-t) T x_{t}$ converges strongly to a point in $\operatorname{Fix}(T)$. If we define a mapping $Q: \Xi_{C} \rightarrow \operatorname{Fix}(T)$ by $Q(f):=s-\lim _{t \rightarrow 0} x_{t}, \forall f \in \Xi_{C}$, then $Q(f)$ solves the VIP:

$$
\begin{array}{r}
\langle(I-f) Q(f), J(Q(f)-p)\rangle \leq 0, \\
\forall f \in \Xi_{C}, p \in \operatorname{Fix}(T) .
\end{array}
$$

Lemma 9 (see [13]). Let $C$ be a nonempty, closed, and convex subset of a strictly convex Banach space X. Let $\left\{T_{n}\right\}_{n=0}^{\infty}$ be a sequence of nonexpansive mappings on $C$. Suppose that $\bigcap_{n=0}^{\infty} \operatorname{Fix}\left(T_{n}\right)$ is nonempty. Let $\left\{\lambda_{n}\right\}$ be a sequence of positive numbers with $\sum_{n=0}^{\infty} \lambda_{n}=1$. Then a mapping $S$ on $C$ defined by $S x=\sum_{n=0}^{\infty} \lambda_{n} T_{n} x$ for $x \in C$ is defined well, nonexpansive, and $\operatorname{Fix}(S)=\bigcap_{n=0}^{\infty} \operatorname{Fix}\left(T_{n}\right)$ holds. 


\section{Implicit Iterative Schemes}

In this section, we introduce our implicit iterative schemes and show the strong convergence theorems. We will use the following useful lemmas in the sequel.

Lemma 10 (see [2, Lemma 2.8]). Let $C$ be a nonempty, closed, and convex subset of a real 2-uniformly smooth Banach space $X$. Let the mapping $B_{i}: C \rightarrow X$ be $\alpha_{i}$-inverse-strongly accretive. Then, one has

$$
\begin{aligned}
& \left\|\left(I-\mu_{i} B_{i}\right) x-\left(I-\mu_{i} B_{i}\right) y\right\|^{2} \\
& \leq\|x-y\|^{2}+2 \mu_{i}\left(\mu_{i} \kappa^{2}-\alpha_{i}\right) \\
& \quad \times\left\|B_{i} x-B_{i} y\right\|^{2}, \quad \forall x, y \in C,
\end{aligned}
$$

for $i=1,2$, where $\mu_{i}>0$. In particular, if $0<\mu_{i} \leq \alpha_{i} / \kappa^{2}$, then $I-\mu_{i} B_{i}$ is nonexpansive for $i=1,2$.

Lemma 11 (see [2, Lemma 2.9]). Let $C$ be a nonempty, closed, and convex subset of a real 2-uniformly smooth Banach space $X$. Let $\Pi_{C}$ be a sunny nonexpansive retraction from $X$ onto $C$. Let the mapping $B_{i}: C \rightarrow X$ be $\alpha_{i}$-inverse-strongly accretive for $i=1,2$. Let $G: C \rightarrow C$ be the mapping defined by

$$
\begin{aligned}
G(x)=\Pi_{C}[ & \Pi_{C}\left(x-\mu_{2} B_{2} x\right) \\
& \left.-\mu_{1} B_{1} \Pi_{C}\left(x-\mu_{2} B_{2} x\right)\right], \quad \forall x \in C .
\end{aligned}
$$

If $0<\mu_{i} \leq \alpha_{i} / \kappa^{2}$ for $i=1,2$, then $G: C \rightarrow C$ is nonexpensive.

Lemma 12 (see [2, Lemma 2.10]). Let C be a nonempty, closed, and convex subset of a real 2-uniformly smooth Banach space $X$. Let $\Pi_{C}$ be a sunny nonexpansive retraction from $X$ onto $C$. Let $B_{1}, B_{2}: C \rightarrow X$ be two nonlinear mappings. For given $x^{*}, y^{*} \in C,\left(x^{*}, y^{*}\right)$ is a solution of GSVI (9) if and only if $x^{*}=\Pi_{C}\left(y^{*}-\mu_{1} B_{1} y^{*}\right)$ where $y^{*}=\Pi_{C}\left(x^{*}-\mu_{2} B_{2} x^{*}\right)$.

Remark 13. By Lemma 12, we observe that

$$
x^{*}=\Pi_{C}\left[\Pi_{C}\left(x^{*}-\mu_{2} B_{2} x^{*}\right)-\mu_{1} B_{1} \Pi_{C}\left(x^{*}-\mu_{2} B_{2} x^{*}\right)\right],
$$

which implies that $x^{*}$ is a fixed point of the mapping $G$.

We now state and prove our first result on the implicit iterative scheme.

Theorem 14. Let $C$ be a nonempty, closed, and convex subset of a uniformly convex and 2-uniformly smooth Banach space $X$. Let $\Pi_{C}$ be a sunny nonexpansive retraction from $X$ onto $C$. Let the mapping $B_{i}: C \rightarrow X$ be $\alpha_{i}$-inverse-strongly accretive for $i=1,2$. Let $f: C \rightarrow C$ be a contraction with coefficient $\rho \in(0,1)$. Let $\left\{S_{n}\right\}_{n=0}^{\infty}$ be an infinite family of nonexpansive mappings of $C$ into itself such that $F=\bigcap_{n=0}^{\infty} \operatorname{Fix}\left(S_{n}\right) \cap \Omega \neq \emptyset$, where $\Omega$ is the fixed point set of the mapping $G=\Pi_{C}(I-$ $\left.\mu_{1} B_{1}\right) \Pi_{C}\left(I-\mu_{2} B_{2}\right)$. For arbitrarily given $x_{0} \in C$, let $\left\{x_{n}\right\}$ be the sequence generated by

$$
\begin{gathered}
y_{n}=\alpha_{n} f\left(y_{n}\right)+\left(1-\alpha_{n}\right) S_{n} G\left(x_{n}\right), \\
x_{n+1}=\beta_{n} x_{n}+\gamma_{n} y_{n}+\delta_{n} S_{n} G\left(y_{n}\right), \quad \forall n \geq 0,
\end{gathered}
$$

where $0<\mu_{i}<\alpha_{i} / \kappa^{2}$ for $i=1,2$ and $\left\{\alpha_{n}\right\},\left\{\beta_{n}\right\},\left\{\gamma_{n}\right\}$, and $\left\{\delta_{n}\right\}$ are the sequences in $(0,1)$ such that $\beta_{n}+\gamma_{n}+\delta_{n}=1, \forall n \geq 0$. Suppose that the following conditions hold:

(i) $\lim _{n \rightarrow \infty} \alpha_{n}=0$ and $\sum_{n=0}^{\infty} \alpha_{n}=\infty$,

(ii) $0<\liminf _{n \rightarrow \infty} \beta_{n} \leq \limsup _{n \rightarrow \infty}\left(\beta_{n}+\gamma_{n}\right)<1$ and $\liminf _{n \rightarrow \infty} \gamma_{n}>0$,

(iii) $\lim _{n \rightarrow \infty}\left|\gamma_{n} /\left(1-\beta_{n}\right)-\gamma_{n-1} /\left(1-\beta_{n-1}\right)\right|=0$.

Assume that $\sum_{n=1}^{\infty} \sup _{x \in D}\left\|S_{n} x-S_{n-1} x\right\|<\infty$ for any bounded subset $D$ of $C$, and let $S$ be a mapping of $C$ into itself defined by $S x=\lim _{n \rightarrow \infty} S_{n} x$ for all $x \in C$. Suppose that $\operatorname{Fix}(S)=$ $\bigcap_{n=0}^{\infty} \operatorname{Fix}\left(S_{n}\right)$. Then $\left\{x_{n}\right\}$ converges strongly to $q \in F$, which solves the following VIP:

$$
\langle q-f(q), J(q-p)\rangle \leq 0, \quad \forall p \in F .
$$

Proof. Take a fixed $p \in F$ arbitrarily. Then by Lemma 12, we know that $p=G(p)$ and $p=S_{n} p$ for all $n \geq 0$. Moreover, by Lemma 11, we have

$$
\begin{aligned}
\left\|y_{n}-p\right\| & \\
= & \left\|\alpha_{n}\left(f\left(y_{n}\right)-p\right)+\left(1-\alpha_{n}\right)\left(S_{n} G\left(x_{n}\right)-p\right)\right\| \\
\leq & \alpha_{n}\left\|f\left(y_{n}\right)-f(p)\right\|+\alpha_{n}\|f(p)-p\| \\
& +\left(1-\alpha_{n}\right)\left\|S_{n} G\left(x_{n}\right)-p\right\| \\
\leq & \alpha_{n} \rho\left\|y_{n}-p\right\|+\alpha_{n}\|f(p)-p\| \\
& +\left(1-\alpha_{n}\right)\left\|G\left(x_{n}\right)-p\right\| \\
\leq & \alpha_{n} \rho\left\|y_{n}-p\right\|+\alpha_{n}\|f(p)-p\| \\
& +\left(1-\alpha_{n}\right)\left\|x_{n}-p\right\|,
\end{aligned}
$$

which hence implies that

$$
\begin{aligned}
& \left\|y_{n}-p\right\| \\
& \leq\left(1-\frac{1-\rho}{1-\alpha_{n} \rho} \alpha_{n}\right)\left\|x_{n}-p\right\| \\
& \quad+\frac{1}{1-\alpha_{n} \rho} \alpha_{n}\|f(p)-p\| \\
& \leq\left\|x_{n}-p\right\|+\frac{\alpha_{n}}{1-\alpha_{n} \rho}\|f(p)-p\| .
\end{aligned}
$$

Thus, from (27), we have

$$
\begin{aligned}
& \left\|x_{n+1}-p\right\| \\
& \quad=\left\|\beta_{n}\left(x_{n}-p\right)+\gamma_{n}\left(y_{n}-p\right)+\delta_{n}\left(S_{n} G\left(y_{n}\right)-p\right)\right\| \\
& \quad \leq \beta_{n}\left\|x_{n}-p\right\|+\gamma_{n}\left\|y_{n}-p\right\|+\delta_{n}\left\|G\left(y_{n}\right)-p\right\| \\
& \quad \leq \beta_{n}\left\|x_{n}-p\right\|+\gamma_{n}\left\|y_{n}-p\right\|+\delta_{n}\left\|y_{n}-p\right\| \\
& \quad=\beta_{n}\left\|x_{n}-p\right\|+\left(1-\beta_{n}\right)\left\|y_{n}-p\right\|
\end{aligned}
$$




$$
\begin{aligned}
\leq & \beta_{n}\left\|x_{n}-p\right\|+\left(1-\beta_{n}\right) \\
\times & \left\{\left(1-\frac{1-\rho}{1-\alpha_{n} \rho} \alpha_{n}\right)\left\|x_{n}-p\right\|\right. \\
& \left.+\frac{1}{1-\alpha_{n} \rho} \alpha_{n}\|f(p)-p\|\right\} \\
= & {\left[1-\frac{\left(1-\beta_{n}\right)(1-\rho)}{1-\alpha_{n} \rho} \alpha_{n}\right]\left\|x_{n}-p\right\| } \\
& +\frac{\left(1-\beta_{n}\right)(1-\rho)}{1-\alpha_{n} \rho} \alpha_{n} \frac{\|f(p)-p\|}{1-\rho} \\
\leq & \max \left\{\left\|x_{0}-p\right\|, \frac{\|f(p)-p\|}{1-\rho}\right\} .
\end{aligned}
$$

It immediately follows that $\left\{x_{n}\right\}$ is bounded, and so are the sequences $\left\{y_{n}\right\},\left\{G\left(x_{n}\right)\right\}$, and $\left\{G\left(y_{n}\right)\right\}$ due to (30) and the nonexpansivity of $G$.

Let us show that $\left\|x_{n+1}-x_{n}\right\| \rightarrow 0$ as $n \rightarrow \infty$. As a matter of fact, from (27), we have

$$
\begin{gathered}
y_{n}=\alpha_{n} f\left(y_{n}\right)+\left(1-\alpha_{n}\right) S_{n} G\left(x_{n}\right), \\
y_{n-1}=\alpha_{n-1} f\left(y_{n-1}\right)+\left(1-\alpha_{n-1}\right) S_{n-1} G\left(x_{n-1}\right), \quad \forall n \geq 1 .
\end{gathered}
$$

Simple calculations show that

$$
\begin{aligned}
y_{n}-y_{n-1}= & \alpha_{n}\left(f\left(y_{n}\right)-f\left(y_{n-1}\right)\right) \\
& +\left(\alpha_{n}-\alpha_{n-1}\right)\left(f\left(y_{n-1}\right)-S_{n-1} G\left(x_{n-1}\right)\right) \\
& +\left(1-\alpha_{n}\right)\left(S_{n} G\left(x_{n}\right)-S_{n-1} G\left(x_{n-1}\right)\right) .
\end{aligned}
$$

It follows that

$$
\begin{aligned}
\| y_{n}- & y_{n-1} \| \\
\leq & \alpha_{n}\left\|f\left(y_{n}\right)-f\left(y_{n-1}\right)\right\|+\left|\alpha_{n}-\alpha_{n-1}\right| \\
& \times\left\|f\left(y_{n-1}\right)-S_{n-1} G\left(x_{n-1}\right)\right\| \\
& +\left(1-\alpha_{n}\right)\left\|S_{n} G\left(x_{n}\right)-S_{n-1} G\left(x_{n-1}\right)\right\| \\
\leq & \alpha_{n} \rho\left\|y_{n}-y_{n-1}\right\|+\left|\alpha_{n}-\alpha_{n-1}\right| \\
& \times\left\|f\left(y_{n-1}\right)-S_{n-1} G\left(x_{n-1}\right)\right\|+\left(1-\alpha_{n}\right) \\
& \times\left(\left\|S_{n} G\left(x_{n}\right)-S_{n} G\left(x_{n-1}\right)\right\|\right. \\
& \left.\quad+\left\|S_{n} G\left(x_{n-1}\right)-S_{n-1} G\left(x_{n-1}\right)\right\|\right) \\
\leq & \alpha_{n} \rho\left\|y_{n}-y_{n-1}\right\|+\left|\alpha_{n}-\alpha_{n-1}\right| \\
& \times\left\|f\left(y_{n-1}\right)-S_{n-1} G\left(x_{n-1}\right)\right\|+\left(1-\alpha_{n}\right) \\
& \times\left(\left\|x_{n}-x_{n-1}\right\|+\left\|S_{n} G\left(x_{n-1}\right)-S_{n-1} G\left(x_{n-1}\right)\right\|\right),
\end{aligned}
$$

which hence yields

$$
\begin{aligned}
& \left\|y_{n}-y_{n-1}\right\| \\
& \leq \frac{1-\alpha_{n}}{1-\alpha_{n} \rho}\left(\left\|x_{n}-x_{n-1}\right\|\right. \\
& \left.\quad+\left\|S_{n} G\left(x_{n-1}\right)-S_{n-1} G\left(x_{n-1}\right)\right\|\right) \\
& \quad+\frac{\left|\alpha_{n}-\alpha_{n-1}\right|}{1-\alpha_{n} \rho}\left\|f\left(y_{n-1}\right)-S_{n-1} G\left(x_{n-1}\right)\right\| \\
& \leq\left\|x_{n}-x_{n-1}\right\|+\left\|S_{n} G\left(x_{n-1}\right)-S_{n-1} G\left(x_{n-1}\right)\right\| \\
& \quad+\frac{\left|\alpha_{n}-\alpha_{n-1}\right|}{1-\alpha_{n} \rho}\left\|f\left(y_{n-1}\right)-S_{n-1} G\left(x_{n-1}\right)\right\| .
\end{aligned}
$$

Now, we write $x_{n}=\beta_{n-1} x_{n-1}+\left(1-\beta_{n-1}\right) z_{n-1}, \forall n \geq 1$, where $z_{n-1}=\left(x_{n}-\beta_{n-1} x_{n-1}\right) /\left(1-\beta_{n-1}\right)$. It follows that for all $n \geq 1$,

$$
\begin{aligned}
z_{n}-z_{n-1}= & \frac{x_{n+1}-\beta_{n} x_{n}}{1-\beta_{n}}-\frac{x_{n}-\beta_{n-1} x_{n-1}}{1-\beta_{n-1}} \\
= & \frac{\gamma_{n} y_{n}+\delta_{n} S_{n} G\left(y_{n}\right)}{1-\beta_{n}} \\
& -\frac{\gamma_{n-1} y_{n-1}+\delta_{n-1} S_{n-1} G\left(y_{n-1}\right)}{1-\beta_{n-1}} \\
= & \frac{\gamma_{n}\left(y_{n}-y_{n-1}\right)+\delta_{n}\left(S_{n} G\left(y_{n}\right)-S_{n-1} G\left(y_{n-1}\right)\right)}{1-\beta_{n}} \\
& +\left(\frac{\gamma_{n}}{1-\beta_{n}}-\frac{\gamma_{n-1}}{1-\beta_{n-1}}\right) y_{n-1} \\
& +\left(\frac{\delta_{n}}{1-\beta_{n}}-\frac{\delta_{n-1}}{1-\beta_{n-1}}\right) S_{n-1} G\left(y_{n-1}\right) .
\end{aligned}
$$

This together with (35) implies that

$$
\begin{aligned}
& \left\|z_{n}-z_{n-1}\right\| \\
& \leq \frac{\left\|\gamma_{n}\left(y_{n}-y_{n-1}\right)+\delta_{n}\left(S_{n} G\left(y_{n}\right)-S_{n-1} G\left(y_{n-1}\right)\right)\right\|}{1-\beta_{n}} \\
& \quad+\left|\frac{\gamma_{n}}{1-\beta_{n}}-\frac{\gamma_{n-1}}{1-\beta_{n-1}}\right|\left\|y_{n-1}\right\| \\
& \quad+\left|\frac{\delta_{n}}{1-\beta_{n}}-\frac{\delta_{n-1}}{1-\beta_{n-1}}\right|\left\|S_{n-1} G\left(y_{n-1}\right)\right\| \\
& \leq\left(\gamma_{n}\left\|y_{n}-y_{n-1}\right\|\right. \\
& \quad+\delta_{n}\left(\left\|S_{n} G\left(y_{n}\right)-S_{n} G\left(y_{n-1}\right)\right\|\right. \\
& \left.\left.\quad+\left\|S_{n} G\left(y_{n-1}\right)-S_{n-1} G\left(y_{n-1}\right)\right\|\right)\right)
\end{aligned}
$$




$$
\begin{aligned}
& \times\left(1-\beta_{n}\right)^{-1} \\
& +\left|\frac{\gamma_{n}}{1-\beta_{n}}-\frac{\gamma_{n-1}}{1-\beta_{n-1}}\right|\left\|y_{n-1}\right\| \\
& +\left|\frac{\gamma_{n}}{1-\beta_{n}}-\frac{\gamma_{n-1}}{1-\beta_{n-1}}\right|\left\|S_{n-1} G\left(y_{n-1}\right)\right\| \\
& \leq \frac{\gamma_{n}\left\|y_{n}-y_{n-1}\right\|+\delta_{n}\left\|y_{n}-y_{n-1}\right\|}{\gamma_{n}+\delta_{n}} \\
& +\frac{\delta_{n}}{\gamma_{n}+\delta_{n}}\left\|S_{n} G\left(y_{n-1}\right)-S_{n-1} G\left(y_{n-1}\right)\right\| \\
& +\left|\frac{\gamma_{n}}{1-\beta_{n}}-\frac{\gamma_{n-1}}{1-\beta_{n-1}}\right|\left(\left\|y_{n-1}\right\|+\left\|S_{n-1} G\left(y_{n-1}\right)\right\|\right) \\
& \leq\left\|y_{n}-y_{n-1}\right\|+\left\|S_{n} G\left(y_{n-1}\right)-S_{n-1} G\left(y_{n-1}\right)\right\| \\
& +\left|\frac{\gamma_{n}}{1-\beta_{n}}-\frac{\gamma_{n-1}}{1-\beta_{n-1}}\right|\left(\left\|y_{n-1}\right\|+\left\|S_{n-1} G\left(y_{n-1}\right)\right\|\right) \\
& \leq\left\|x_{n}-x_{n-1}\right\|+\left\|S_{n} G\left(x_{n-1}\right)-S_{n-1} G\left(x_{n-1}\right)\right\| \\
& +\frac{\left|\alpha_{n}-\alpha_{n-1}\right|}{1-\alpha_{n} \rho}\left\|f\left(y_{n-1}\right)-S_{n-1} G\left(x_{n-1}\right)\right\| \\
& +\left\|S_{n} G\left(y_{n-1}\right)-S_{n-1} G\left(y_{n-1}\right)\right\| \\
& +\left|\frac{\gamma_{n}}{1-\beta_{n}}-\frac{\gamma_{n-1}}{1-\beta_{n-1}}\right|\left(\left\|y_{n-1}\right\|+\left\|S_{n-1} G\left(y_{n-1}\right)\right\|\right) \\
& \leq\left\|x_{n}-x_{n-1}\right\|+\left\|S_{n} G\left(x_{n-1}\right)-S_{n-1} G\left(x_{n-1}\right)\right\| \\
& +\frac{\left|\alpha_{n}-\alpha_{n-1}\right|}{1-\alpha_{n} \rho} M+\left\|S_{n} G\left(y_{n-1}\right)-S_{n-1} G\left(y_{n-1}\right)\right\| \\
& +\left|\frac{\gamma_{n}}{1-\beta_{n}}-\frac{\gamma_{n-1}}{1-\beta_{n-1}}\right| M \\
& =\left\|x_{n}-x_{n-1}\right\| \\
& +M\left(\frac{1}{1-\alpha_{n} \rho}\left|\alpha_{n}-\alpha_{n-1}\right|+\left|\frac{\gamma_{n}}{1-\beta_{n}}-\frac{\gamma_{n-1}}{1-\beta_{n-1}}\right|\right) \\
& +\left\|S_{n} G\left(x_{n-1}\right)-S_{n-1} G\left(x_{n-1}\right)\right\| \\
& +\left\|S_{n} G\left(y_{n-1}\right)-S_{n-1} G\left(y_{n-1}\right)\right\| \text {, }
\end{aligned}
$$

where $\sup _{n \geq 0}\left\{\left\|f\left(y_{n}\right)\right\|+\left\|S_{n} G\left(x_{n}\right)\right\|+\left\|y_{n}\right\|+\left\|S_{n} G\left(y_{n}\right)\right\|\right\} \leq M$ for some $M>0$. So, from $\alpha_{n} \rightarrow 0$, condition (iii), and the assumption on $\left\{S_{n}\right\}$, it immediately follows that

$$
\limsup _{n \rightarrow \infty}\left(\left\|z_{n}-z_{n-1}\right\|-\left\|x_{n}-x_{n-1}\right\|\right) \leq 0
$$

In terms of condition (ii) and Lemma 4, we get

$$
\lim _{n \rightarrow \infty}\left\|z_{n}-x_{n}\right\|=0
$$

Hence we obtain

$$
\lim _{n \rightarrow \infty}\left\|x_{n+1}-x_{n}\right\|=\lim _{n \rightarrow \infty}\left(1-\beta_{n}\right)\left\|z_{n}-x_{n}\right\|=0 .
$$

Next we show that $\left\|x_{n}-G\left(x_{n}\right)\right\| \rightarrow 0$ as $n \rightarrow \infty$.

For simplicity, put $q=\Pi_{C}\left(p-\mu_{2} B_{2} p\right), u_{n}=\Pi_{C}\left(x_{n}-\right.$ $\left.\mu_{2} B_{2} x_{n}\right)$, and $v_{n}=\Pi_{C}\left(u_{n}-\mu_{1} B_{1} u_{n}\right)$. Then $v_{n}=G\left(x_{n}\right)$. From Lemma 10, we have

$$
\begin{aligned}
\left\|u_{n}-q\right\|^{2} & =\left\|\Pi_{C}\left(x_{n}-\mu_{2} B_{2} x_{n}\right)-\Pi_{C}\left(p-\mu_{2} B_{2} p\right)\right\|^{2} \\
& \leq\left\|x_{n}-p-\mu_{2}\left(B_{2} x_{n}-B_{2} p\right)\right\|^{2} \\
& \leq\left\|x_{n}-p\right\|^{2}-2 \mu_{2}\left(\alpha_{2}-\kappa^{2} \mu_{2}\right)\left\|B_{2} x_{n}-B_{2} p\right\|^{2}, \\
\left\|v_{n}-p\right\|^{2} & =\left\|\Pi_{C}\left(u_{n}-\mu_{1} B_{1} u_{n}\right)-\Pi_{C}\left(q-\mu_{1} B_{1} q\right)\right\|^{2} \\
& \leq\left\|u_{n}-q-\mu_{1}\left(B_{1} u_{n}-B_{1} q\right)\right\|^{2} \\
& \leq\left\|u_{n}-q\right\|^{2}-2 \mu_{1}\left(\alpha_{1}-\kappa^{2} \mu_{1}\right)\left\|B_{1} u_{n}-B_{1} q\right\|^{2} .
\end{aligned}
$$

Substituting (41) into (42), we obtain

$$
\begin{aligned}
\left\|v_{n}-p\right\|^{2} \leq & \left\|x_{n}-p\right\|^{2}-2 \mu_{2}\left(\alpha_{2}-\kappa^{2} \mu_{2}\right)\left\|B_{2} x_{n}-B_{2} p\right\|^{2} \\
& -2 \mu_{1}\left(\alpha_{1}-\kappa^{2} \mu_{1}\right)\left\|B_{1} u_{n}-B_{1} q\right\|^{2} .
\end{aligned}
$$

According to Lemma 3, we have from (27)

$$
\begin{aligned}
& \left\|y_{n}-p\right\|^{2} \\
& =\| \alpha_{n}\left(f\left(y_{n}\right)-f(p)\right)+\left(1-\alpha_{n}\right)\left(S_{n} v_{n}-p\right) \\
& +\alpha_{n}(f(p)-p) \|^{2} \\
& \leq\left\|\alpha_{n}\left(f\left(y_{n}\right)-f(p)\right)+\left(1-\alpha_{n}\right)\left(S_{n} v_{n}-p\right)\right\|^{2} \\
& +2 \alpha_{n}\left\langle f(p)-p, J\left(y_{n}-p\right)\right\rangle \\
& \leq \alpha_{n}\left\|f\left(y_{n}\right)-f(p)\right\|^{2}+\left(1-\alpha_{n}\right)\left\|S_{n} v_{n}-p\right\|^{2} \\
& +2 \alpha_{n}\left\langle f(p)-p, J\left(y_{n}-p\right)\right\rangle \\
& \leq \alpha_{n} \rho\left\|y_{n}-p\right\|^{2}+\left(1-\alpha_{n}\right)\left\|v_{n}-p\right\|^{2} \\
& +2 \alpha_{n}\|f(p)-p\|\left\|y_{n}-p\right\|,
\end{aligned}
$$

which hence yields

$$
\begin{aligned}
\left\|y_{n}-p\right\|^{2} & \\
\leq & \left(1-\frac{1-\rho}{1-\alpha_{n} \rho} \alpha_{n}\right)\left\|v_{n}-p\right\|^{2} \\
& +\frac{2 \alpha_{n}}{1-\alpha_{n} \rho}\|f(p)-p\|\left\|y_{n}-p\right\| .
\end{aligned}
$$


This together with (43) and the convexity of $\|\cdot\|^{2}$, we have

$$
\begin{aligned}
& \left\|x_{n+1}-p\right\|^{2} \\
& =\left\|\beta_{n}\left(x_{n}-p\right)+\gamma_{n}\left(y_{n}-p\right)+\delta_{n}\left(S_{n} G\left(y_{n}\right)-p\right)\right\|^{2} \\
& \leq \beta_{n}\left\|x_{n}-p\right\|^{2}+\gamma_{n}\left\|y_{n}-p\right\|^{2}+\delta_{n}\left\|S_{n} G\left(y_{n}\right)-p\right\|^{2} \\
& \leq \beta_{n}\left\|x_{n}-p\right\|^{2}+\gamma_{n}\left\|y_{n}-p\right\|^{2}+\delta_{n}\left\|y_{n}-p\right\|^{2} \\
& =\beta_{n}\left\|x_{n}-p\right\|^{2}+\left(1-\beta_{n}\right)\left\|y_{n}-p\right\|^{2} \\
& \leq \beta_{n}\left\|x_{n}-p\right\|^{2}+\left(1-\beta_{n}\right) \\
& \times\left\{\left(1-\frac{1-\rho}{1-\alpha_{n} \rho} \alpha_{n}\right)\left\|v_{n}-p\right\|^{2}\right. \\
& \left.+\frac{2 \alpha_{n}}{1-\alpha_{n} \rho}\|f(p)-p\|\left\|y_{n}-p\right\|\right\} \\
& \leq \beta_{n}\left\|x_{n}-p\right\|^{2}+\left(1-\beta_{n}\right) \\
& \times\left(1-\frac{1-\rho}{1-\alpha_{n} \rho} \alpha_{n}\right)\left\|v_{n}-p\right\|^{2}+\alpha_{n} M_{1} \\
& \leq \beta_{n}\left\|x_{n}-p\right\|^{2}+\left(1-\beta_{n}\right)\left(1-\frac{1-\rho}{1-\alpha_{n} \rho} \alpha_{n}\right) \\
& \times\left[\left\|x_{n}-p\right\|^{2}-2 \mu_{2}\left(\alpha_{2}-\kappa^{2} \mu_{2}\right)\left\|B_{2} x_{n}-B_{2} p\right\|^{2}\right. \\
& \left.-2 \mu_{1}\left(\alpha_{1}-\kappa^{2} \mu_{1}\right)\left\|B_{1} u_{n}-B_{1} q\right\|^{2}\right]+\alpha_{n} M_{1} \\
& =\left(1-\frac{\left(1-\beta_{n}\right)(1-\rho)}{1-\alpha_{n} \rho} \alpha_{n}\right)\left\|x_{n}-p\right\|^{2}-2\left(1-\beta_{n}\right) \\
& \times\left(1-\frac{1-\rho}{1-\alpha_{n} \rho} \alpha_{n}\right) \\
& \times\left[\mu_{2}\left(\alpha_{2}-\kappa^{2} \mu_{2}\right)\left\|B_{2} x_{n}-B_{2} p\right\|^{2}\right. \\
& \left.+\mu_{1}\left(\alpha_{1}-\kappa^{2} \mu_{1}\right)\left\|B_{1} u_{n}-B_{1} q\right\|^{2}\right]+\alpha_{n} M_{1} \\
& \leq\left\|x_{n}-p\right\|^{2}-2\left(1-\beta_{n}\right)\left(1-\frac{1-\rho}{1-\alpha_{n} \rho} \alpha_{n}\right) \\
& \times\left[\mu_{2}\left(\alpha_{2}-\kappa^{2} \mu_{2}\right)\left\|B_{2} x_{n}-B_{2} p\right\|^{2}\right. \\
& \left.+\mu_{1}\left(\alpha_{1}-\kappa^{2} \mu_{1}\right)\left\|B_{1} u_{n}-B_{1} q\right\|^{2}\right]+\alpha_{n} M_{1},
\end{aligned}
$$

where $\sup _{n \geq 0}\left\{2\left(1-\beta_{n}\right) /\left(1-\alpha_{n} \rho\right)\|f(p)-p\|\left\|y_{n}-p\right\|\right\} \leq M_{1}$ for some $M_{1}>0$. So, it follows that

$$
\begin{aligned}
& 2\left(1-\beta_{n}\right)\left(1-\frac{1-\rho}{1-\alpha_{n} \rho} \alpha_{n}\right) \\
& \times\left[\mu_{2}\left(\alpha_{2}-\kappa^{2} \mu_{2}\right)\left\|B_{2} x_{n}-B_{2} p\right\|^{2}\right. \\
& \left.\quad+\mu_{1}\left(\alpha_{1}-\kappa^{2} \mu_{1}\right)\left\|B_{1} \mathrm{u}_{n}-B_{1} q\right\|^{2}\right]
\end{aligned}
$$

$$
\begin{aligned}
& \leq\left\|x_{n}-p\right\|^{2}-\left\|x_{n+1}-p\right\|^{2}+\alpha_{n} M_{1} \\
& \leq\left(\left\|x_{n}-p\right\|+\left\|x_{n+1}-p\right\|\right)\left\|x_{n}-x_{n+1}\right\|+\alpha_{n} M_{1} .
\end{aligned}
$$

Since $0<\mu_{i}<\alpha_{i} / \kappa^{2}$ for $i=1,2$, from conditions (i), (ii), and (40), we obtain

$$
\lim _{n \rightarrow \infty}\left\|B_{2} x_{n}-B_{2} p\right\|=0, \quad \lim _{n \rightarrow \infty}\left\|B_{1} u_{n}-B_{1} q\right\|=0 .
$$

Utilizing [14, Proposition 1] and Lemma 5, we have

$$
\begin{aligned}
&\left\|u_{n}-q\right\|^{2} \\
&=\left\|\Pi_{C}\left(x_{n}-\mu_{2} B_{2} x_{n}\right)-\Pi_{C}\left(p-\mu_{2} B_{2} p\right)\right\|^{2} \\
& \leq\left\langle x_{n}-\mu_{2} B_{2} x_{n}-\left(p-\mu_{2} B_{2} p\right), J\left(u_{n}-q\right)\right\rangle \\
&=\left\langle x_{n}-p, J\left(u_{n}-q\right)\right\rangle \\
&+\mu_{2}\left\langle B_{2} p-B_{2} x_{n}, J\left(u_{n}-q\right)\right\rangle \\
& \leq \frac{1}{2}\left[\left\|x_{n}-p\right\|^{2}+\left\|u_{n}-q\right\|^{2}-g_{1}\left(\left\|x_{n}-u_{n}-(p-q)\right\|\right)\right] \\
&+\mu_{2}\left\|B_{2} p-B_{2} x_{n}\right\|\left\|u_{n}-q\right\|,
\end{aligned}
$$

which implies that

$$
\begin{aligned}
\left\|u_{n}-q\right\|^{2} \leq & \left\|x_{n}-p\right\|^{2}-g_{1}\left(\left\|x_{n}-u_{n}-(p-q)\right\|\right) \\
& +2 \mu_{2}\left\|B_{2} p-B_{2} x_{n}\right\|\left\|u_{n}-q\right\| .
\end{aligned}
$$

In the same way, we derive

$$
\begin{aligned}
&\left\|v_{n}-p\right\|^{2} \\
&=\left\|\Pi_{C}\left(u_{n}-\mu_{1} B_{1} u_{n}\right)-\Pi_{C}\left(q-\mu_{1} B_{1} q\right)\right\|^{2} \\
& \leq\left\langle u_{n}-\mu_{1} B_{1} u_{n}-\left(q-\mu_{1} B_{1} q\right), J\left(v_{n}-p\right)\right\rangle \\
&=\left\langle u_{n}-q, J\left(v_{n}-p\right)\right\rangle+\mu_{1}\left\langle B_{1} q-B_{1} u_{n}, J\left(v_{n}-p\right)\right\rangle \\
& \leq \frac{1}{2}\left[\left\|u_{n}-q\right\|^{2}+\left\|v_{n}-p\right\|^{2}\right. \\
&\left.\quad-g_{2}\left(\left\|u_{n}-v_{n}+(p-q)\right\|\right)\right] \\
&+\mu_{1}\left\|B_{1} q-B_{1} u_{n}\right\|\left\|v_{n}-p\right\|,
\end{aligned}
$$

which implies that

$$
\begin{aligned}
\left\|v_{n}-p\right\|^{2} \leq & \left\|u_{n}-q\right\|^{2}-g_{2}\left(\left\|u_{n}-v_{n}+(p-q)\right\|\right) \\
& +2 \mu_{1}\left\|B_{1} q-B_{1} u_{n}\right\|\left\|v_{n}-p\right\| .
\end{aligned}
$$

Substituting (50) into (52), we get

$$
\begin{aligned}
\left\|v_{n}-p\right\|^{2} \leq & \left\|x_{n}-p\right\|^{2}-g_{1}\left(\left\|x_{n}-u_{n}-(p-q)\right\|\right) \\
& -g_{2}\left(\left\|u_{n}-v_{n}+(p-q)\right\|\right) \\
& +2 \mu_{2}\left\|B_{2} p-B_{2} x_{n}\right\|\left\|u_{n}-q\right\| \\
& +2 \mu_{1}\left\|B_{1} q-B_{1} u_{n}\right\|\left\|v_{n}-p\right\| .
\end{aligned}
$$


From (46) and (53), we have

$$
\begin{aligned}
\left\|x_{n+1}-p\right\|^{2} \leq & \alpha_{n} M_{1}+\beta_{n}\left\|x_{n}-p\right\|^{2} \\
& +\left(1-\beta_{n}\right)\left(1-\frac{1-\rho}{1-\alpha_{n} \rho} \alpha_{n}\right) \\
& \times\left[\left\|x_{n}-p\right\|^{2}-g_{1}\left(\left\|x_{n}-u_{n}-(p-q)\right\|\right)\right. \\
& \quad-g_{2}\left(\left\|u_{n}-v_{n}+(p-q)\right\|\right) \\
& +2 \mu_{2}\left\|B_{2} p-B_{2} x_{n}\right\|\left\|u_{n}-q\right\| \\
& \left.+2 \mu_{1}\left\|B_{1} q-B_{1} u_{n}\right\|\left\|v_{n}-p\right\|\right] \\
\leq & \alpha_{n} M_{1}+\left(1-\frac{\left(1-\beta_{n}\right)(1-\rho)}{1-\alpha_{n} \rho} \alpha_{n}\right)\left\|x_{n}-p\right\|^{2} \\
& +\left(1-\beta_{n}\right)\left(1-\frac{1-\rho}{1-\alpha_{n} \rho} \alpha_{n}\right) \\
& +2 \mu_{2}\left\|B_{2} p-B_{2} x_{n}\right\|\left\|u_{n}-q\right\| \\
& \times\left[g_{1}\left(\left\|x_{n}-u_{n}-(p-q)\right\|\right)\right. \\
& \times\left(1-\frac{1}{1-\beta_{1}}\right) \\
& \left.+g_{2}\left(\left\|u_{n}-v_{n}+(p-q)\right\|\right)\right] \\
+ & +2 \mu_{2}\left\|B_{2} p-B_{2} x_{n}\right\|\left\|u_{n}-q\right\| \\
+ & +\mu_{1}\left\|B_{1} q-B_{1} u_{n}\right\|\left\|v_{n}-p\right\|
\end{aligned}
$$

which implies that

$$
\begin{aligned}
\left(1-\beta_{n}\right) & \left(1-\frac{1-\rho}{1-\alpha_{n} \rho} \alpha_{n}\right) \\
& \times\left[g_{1}\left(\left\|x_{n}-u_{n}-(p-q)\right\|\right)\right. \\
& \left.+g_{2}\left(\left\|u_{n}-v_{n}+(p-q)\right\|\right)\right] \\
\leq & \alpha_{n} M_{1}+\left\|x_{n}-p\right\|^{2}-\left\|x_{n+1}-p\right\|^{2} \\
& +2 \mu_{2}\left\|B_{2} p-B_{2} x_{n}\right\|\left\|u_{n}-q\right\| \\
& +2 \mu_{1}\left\|B_{1} q-B_{1} u_{n}\right\|\left\|v_{n}-p\right\| \\
\leq & \alpha_{n} M_{1}+\left(\left\|x_{n}-p\right\|+\left\|x_{n+1}-p\right\|\right)\left\|x_{n}-x_{n+1}\right\| \\
& +2 \mu_{2}\left\|B_{2} p-B_{2} x_{n}\right\|\left\|u_{n}-q\right\| \\
& +2 \mu_{1}\left\|B_{1} q-B_{1} u_{n}\right\|\left\|v_{n}-p\right\| .
\end{aligned}
$$

Utilizing conditions (i), (ii), from (40) and (48), we have

$$
\begin{aligned}
& \lim _{n \rightarrow \infty} g_{1}\left(\left\|x_{n}-u_{n}-(p-q)\right\|\right)=0, \\
& \lim _{n \rightarrow \infty} g_{2}\left(\left\|u_{n}-v_{n}+(p-q)\right\|\right)=0 .
\end{aligned}
$$

Utilizing the properties of $g_{1}$ and $g_{2}$, we deduce that

$$
\begin{aligned}
& \lim _{n \rightarrow \infty}\left\|x_{n}-u_{n}-(p-q)\right\|=0, \\
& \lim _{n \rightarrow \infty}\left\|u_{n}-v_{n}+(p-q)\right\|=0 .
\end{aligned}
$$

From (57), we obtain

$$
\begin{aligned}
\left\|x_{n}-v_{n}\right\| \leq & \left\|x_{n}-u_{n}-(p-q)\right\| \\
& +\left\|u_{n}-v_{n}+(p-q)\right\| \longrightarrow 0 \quad \text { as } n \rightarrow \infty
\end{aligned}
$$

That is,

$$
\lim _{n \rightarrow \infty}\left\|x_{n}-G\left(x_{n}\right)\right\|=0 .
$$

On the other hand, since $\left\{y_{n}\right\}$ and $\left\{S_{n} G\left(y_{n}\right)\right\}$ are bounded, by Lemma 6 , there exists a continuous strictly increasing function $g_{3}:[0, \infty) \rightarrow[0, \infty), g_{3}(0)=0$ such that for $p \in F$

$$
\begin{aligned}
\left\|x_{n+1}-p\right\|^{2} & \left\|\beta_{n}\left(x_{n}-p\right)+\gamma_{n}\left(y_{n}-p\right)+\delta_{n}\left(S_{n} G\left(y_{n}\right)-p\right)\right\|^{2} \\
= & \|\left(\gamma_{n}+\delta_{n}\right)\left[\frac{\gamma_{n}}{\gamma_{n}+\delta_{n}}\left(y_{n}-p\right)\right. \\
& \left.\quad+\frac{\delta_{n}}{\gamma_{n}+\delta_{n}}\left(S_{n} G\left(y_{n}\right)-p\right)\right]+\beta_{n}\left(x_{n}-p\right) \|^{2} \\
\leq & \left(\gamma_{n}+\delta_{n}\right)\left\|\frac{\gamma_{n}}{\gamma_{n}+\delta_{n}}\left(y_{n}-p\right)+\frac{\delta_{n}}{\gamma_{n}+\delta_{n}}\left(S_{n} G\left(y_{n}\right)-p\right)\right\|^{2} \\
& +\beta_{n}\left\|x_{n}-p\right\|^{2} \\
\leq & \left(\gamma_{n}+\delta_{n}\right)\left[\frac{\gamma_{n}}{\gamma_{n}+\delta_{n}}\left\|y_{n}-p\right\|^{2}+\frac{\delta_{n}}{\gamma_{n}+\delta_{n}}\left\|S_{n} G\left(y_{n}\right)-p\right\|^{2}\right. \\
& \quad\left(1-\beta_{n}\right)\left\|y_{n}-p\right\|^{2}-\frac{\gamma_{n} \delta_{n}}{\gamma_{n}+\delta_{n}} \\
& +\beta_{n}\left\|x_{n}-p\right\|^{2} \\
\leq & \gamma_{n}\left\|y_{n}-p\right\|^{2}+\delta_{n}\left\|y_{n}-p\right\|^{2} \\
& \left.\quad-g_{n}\left(\left\|y_{n}-S_{n} G\left(y_{n}\right)\right\|\right)+\beta_{n}\left\|x_{n}-p\right\|^{2} g_{3}\left(\left\|y_{n}-S_{n} G\left(y_{n}\right)\right\|\right)\right]
\end{aligned}
$$


which together with (30) implies that

$$
\begin{aligned}
\| x_{n+1}- & p \|^{2} \\
\leq & \left(1-\beta_{n}\right)\left(\left\|x_{n}-p\right\|+\frac{\alpha_{n}}{1-\alpha_{n} \rho}\|f(p)-p\|\right)^{2} \\
& \quad-\frac{\gamma_{n} \delta_{n}}{\gamma_{n}+\delta_{n}} g_{3}\left(\left\|y_{n}-S_{n} G\left(y_{n}\right)\right\|\right)+\beta_{n}\left\|x_{n}-p\right\|^{2} \\
\leq & \left(\left\|x_{n}-p\right\|+\frac{\alpha_{n}}{1-\alpha_{n} \rho}\|f(p)-p\|\right)^{2} \\
& \quad-\frac{\gamma_{n} \delta_{n}}{\gamma_{n}+\delta_{n}} g_{3}\left(\left\|y_{n}-S_{n} G\left(y_{n}\right)\right\|\right) .
\end{aligned}
$$

It immediately follows that

$$
\begin{aligned}
\gamma_{n} \delta_{n} g_{3} & \left(\left\|y_{n}-S_{n} G\left(y_{n}\right)\right\|\right) \\
\leq & \frac{\gamma_{n} \delta_{n}}{\gamma_{n}+\delta_{n}} g_{3}\left(\left\|y_{n}-S_{n} G\left(y_{n}\right)\right\|\right) \\
\leq & \left(\left\|x_{n}-p\right\|+\frac{\alpha_{n}}{1-\alpha_{n} \rho}\|f(p)-p\|\right)^{2} \\
& -\left\|x_{n+1}-p\right\|^{2} \\
\leq & \left(\left\|x_{n}-p\right\|+\left\|x_{n+1}-p\right\|\right. \\
& \left.\quad+\frac{\alpha_{n}}{1-\alpha_{n} \rho}\|f(p)-p\|\right) \\
& \times\left(\left\|x_{n}-x_{n+1}\right\|+\frac{\alpha_{n}}{1-\alpha_{n} \rho}\|f(p)-p\|\right) .
\end{aligned}
$$

According to condition (ii), we get

$$
\begin{aligned}
\liminf _{n \rightarrow \infty} \delta_{n} & =\liminf _{n \rightarrow \infty}\left(1-\beta_{n}-\gamma_{n}\right) \\
& =1-\limsup _{n \rightarrow \infty}\left(\beta_{n}+\gamma_{n}\right)>0 .
\end{aligned}
$$

Since $\alpha_{n} \rightarrow 0,\left\|x_{n+1}-x_{n}\right\| \rightarrow 0$, and $\liminf _{n \rightarrow \infty} \gamma_{n}>0$, we conclude that

$$
\lim _{n \rightarrow \infty} g_{3}\left(\left\|y_{n}-S_{n} G\left(y_{n}\right)\right\|\right)=0
$$

Utilizing the property of $g_{3}$, we have

$$
\lim _{n \rightarrow \infty}\left\|y_{n}-S_{n} G\left(y_{n}\right)\right\|=0
$$

We note that

$$
\begin{aligned}
x_{n+1}-x_{n}+x_{n}-y_{n} & =x_{n+1}-y_{n} \\
& =\beta_{n}\left(x_{n}-y_{n}\right)+\delta_{n}\left(S_{n} G\left(y_{n}\right)-y_{n}\right) .
\end{aligned}
$$

So,

$$
\begin{aligned}
\left(1-\beta_{n}\right) & \left\|x_{n}-y_{n}\right\| \\
= & \left\|\delta_{n}\left(S_{n} G\left(y_{n}\right)-y_{n}\right)-\left(x_{n+1}-x_{n}\right)\right\| \\
\leq & \delta_{n}\left\|S_{n} G\left(y_{n}\right)-y_{n}\right\|+\left\|x_{n+1}-x_{n}\right\| \\
\leq & \left\|S_{n} G\left(y_{n}\right)-y_{n}\right\| \\
& +\left\|x_{n+1}-x_{n}\right\| \longrightarrow 0 \quad \text { as } n \longrightarrow \infty .
\end{aligned}
$$

That is,

$$
\lim _{n \rightarrow \infty}\left\|x_{n}-y_{n}\right\|=0 \text {. }
$$

We observe that

$$
\begin{aligned}
\| G\left(x_{n}\right) & -S_{n} G\left(x_{n}\right) \| \\
\leq & \left\|G\left(x_{n}\right)-x_{n}\right\|+\left\|x_{n}-y_{n}\right\|+\left\|y_{n}-S_{n} G\left(y_{n}\right)\right\| \\
& \quad+\left\|S_{n} G\left(y_{n}\right)-S_{n} G\left(x_{n}\right)\right\| \\
\leq & \left\|G\left(x_{n}\right)-x_{n}\right\|+2\left\|x_{n}-y_{n}\right\|+\left\|y_{n}-S_{n} G\left(y_{n}\right)\right\| .
\end{aligned}
$$

Thus, from (59)-(68), we obtain that

$$
\lim _{n \rightarrow \infty}\left\|G\left(x_{n}\right)-S_{n} G\left(x_{n}\right)\right\|=0 .
$$

By (70) and Lemma 7, we have

$$
\begin{aligned}
\left\|S G\left(x_{n}\right)-G\left(x_{n}\right)\right\| & \\
\leq & \left\|S G\left(x_{n}\right)-S_{n} G\left(x_{n}\right)\right\| \\
& +\left\|S_{n} G\left(x_{n}\right)-G\left(x_{n}\right)\right\| \longrightarrow 0 \text { as } n \longrightarrow \infty .
\end{aligned}
$$

In terms of (59) and (71), we have

$$
\begin{aligned}
\left\|x_{n}-S x_{n}\right\| \leq & \left\|x_{n}-G\left(x_{n}\right)\right\|+\left\|G\left(x_{n}\right)-S G\left(x_{n}\right)\right\| \\
& +\left\|S G\left(x_{n}\right)-S x_{n}\right\| \\
\leq & 2\left\|x_{n}-G\left(x_{n}\right)\right\| \\
& +\left\|G\left(x_{n}\right)-S G\left(x_{n}\right)\right\| \longrightarrow 0 \text { as } n \longrightarrow \infty .
\end{aligned}
$$

Define a mapping $W x=(1-\theta) S x+\theta G(x)$, where $\theta \in(0,1)$ is a constant. Then by Lemma 9, we have that $\operatorname{Fix}(W)=\operatorname{Fix}(S) \cap$ $\operatorname{Fix}(G)=F$. We observe that

$$
\begin{aligned}
\left\|x_{n}-W x_{n}\right\| & =\left\|(1-\theta)\left(x_{n}-S x_{n}\right)+\theta\left(x_{n}-G\left(x_{n}\right)\right)\right\| \\
& \leq(1-\theta)\left\|x_{n}-S x_{n}\right\|+\theta\left\|x_{n}-G\left(x_{n}\right)\right\| .
\end{aligned}
$$

From (59) and (72), we obtain

$$
\lim _{n \rightarrow \infty}\left\|x_{n}-W x_{n}\right\|=0 .
$$

Now, we claim that

$$
\limsup _{n \rightarrow \infty}\left\langle f(q)-q, J\left(x_{n}-q\right)\right\rangle \leq 0,
$$


where $q=s-\lim _{t \rightarrow 0} x_{t}$ with $x_{t}$ being the fixed point of the contraction

$$
x \longmapsto t f(x)+(1-t) W x .
$$

Then $x_{t}$ solves the fixed point equation $x_{t}=t f\left(x_{t}\right)+(1-$ $t) W x_{t}$. Thus we have

$$
\left\|x_{t}-x_{n}\right\|=\left\|(1-t)\left(W x_{t}-x_{n}\right)+t\left(f\left(x_{t}\right)-x_{n}\right)\right\| .
$$

By Lemma 3, we conclude that

$$
\begin{aligned}
&\left\|x_{t}-x_{n}\right\|^{2} \\
&=\left\|(1-t)\left(W x_{t}-x_{n}\right)+t\left(f\left(x_{t}\right)-x_{n}\right)\right\|^{2} \\
& \leq(1-t)^{2}\left\|W x_{t}-x_{n}\right\|^{2} \\
&+2 t\left\langle f\left(x_{t}\right)-x_{n}, J\left(x_{t}-x_{n}\right)\right\rangle \\
& \leq(1-t)^{2}\left(\left\|W x_{t}-W x_{n}\right\|+\left\|W x_{n}-x_{n}\right\|\right)^{2} \\
&+2 t\left\langle f\left(x_{t}\right)-x_{n}, J\left(x_{t}-x_{n}\right)\right\rangle \\
& \leq(1-t)^{2}\left(\left\|x_{t}-x_{n}\right\|+\left\|W x_{n}-x_{n}\right\|\right)^{2} \\
&+2 t\left\langle f\left(x_{t}\right)-x_{n}, J\left(x_{t}-x_{n}\right)\right\rangle \\
&=(1-t)^{2}\left[\left\|x_{t}-x_{n}\right\|^{2}+2\left\|x_{t}-x_{n}\right\|\right. \\
&\left.\quad \times\left\|W x_{n}-x_{n}\right\|+\left\|W x_{n}-x_{n}\right\|^{2}\right] \\
&+2 t\left\langle f\left(x_{t}\right)-x_{t}, J\left(x_{t}-x_{n}\right)\right\rangle+2 t\left\|x_{t}-x_{n}\right\|^{2}, \\
&+2 t\left\langle f\left(x_{t}\right)-x_{t}, J\left(x_{t}-x_{n}\right)\right\rangle \\
&\left(1-2 t+x_{t}-x_{n}, J\left(x_{t}-x_{n}\right)\right\rangle \\
&+x_{t}-x_{n} \|^{2}+f_{n}(t)
\end{aligned}
$$

where

$$
\begin{aligned}
f_{n}(t)= & (1-t)^{2}\left(2\left\|x_{t}-x_{n}\right\|+\left\|x_{n}-W x_{n}\right\|\right) \\
& \times\left\|x_{n}-W x_{n}\right\| \longrightarrow 0, \quad \text { as } n \longrightarrow \infty .
\end{aligned}
$$

It follows from (78) that

$$
\left\langle x_{t}-f\left(x_{t}\right), J\left(x_{t}-x_{n}\right)\right\rangle \leq \frac{t}{2}\left\|x_{t}-x_{n}\right\|^{2}+\frac{1}{2 t} f_{n}(t) .
$$

Letting $n \rightarrow \infty$ in (80) and noticing (79), we derive

$$
\limsup _{n \rightarrow \infty}\left\langle x_{t}-f\left(x_{t}\right), J\left(x_{t}-x_{n}\right)\right\rangle \leq \frac{t}{2} M_{2}
$$

where $M_{2}>0$ is a constant such that $\left\|x_{t}-x_{n}\right\|^{2} \leq M_{2}$ for all $t \in(0,1)$ and $n \geq 0$. Taking $t \rightarrow 0$ in (81), we have

$$
\limsup \limsup _{t \rightarrow 0}\left\langle x_{t}-f\left(x_{t}\right), J\left(x_{t}-x_{n}\right)\right\rangle \leq 0 \text {. }
$$

On the other hand, we have

$$
\begin{aligned}
\langle f(q) & \left.-q, J\left(x_{n}-q\right)\right\rangle \\
= & \left\langle f(q)-q, J\left(x_{n}-q\right)\right\rangle-\left\langle f(q)-q, J\left(x_{n}-x_{t}\right)\right\rangle \\
& +\left\langle f(q)-q, J\left(x_{n}-x_{t}\right)\right\rangle-\left\langle f(q)-x_{t}, J\left(x_{n}-x_{t}\right)\right\rangle \\
& +\left\langle f(q)-x_{t}, J\left(x_{n}-x_{t}\right)\right\rangle-\left\langle f\left(x_{t}\right)-x_{t}, J\left(x_{n}-x_{t}\right)\right\rangle \\
& +\left\langle f\left(x_{t}\right)-x_{t}, J\left(x_{n}-x_{t}\right)\right\rangle \\
= & \left\langle f(q)-q, J\left(x_{n}-q\right)-J\left(x_{n}-x_{t}\right)\right\rangle \\
& +\left\langle x_{t}-q, J\left(x_{n}-x_{t}\right)\right\rangle+\left\langle f(q)-f\left(x_{t}\right), J\left(x_{n}-x_{t}\right)\right\rangle \\
& +\left\langle f\left(x_{t}\right)-x_{t}, J\left(x_{n}-x_{t}\right)\right\rangle .
\end{aligned}
$$

It follows that

$$
\begin{aligned}
\limsup _{n \rightarrow \infty} & \left\langle f(q)-q, J\left(x_{n}-q\right)\right\rangle \\
\leq & \limsup _{n \rightarrow \infty}\left\langle f(q)-q, J\left(x_{n}-q\right)-J\left(x_{n}-x_{t}\right)\right\rangle \\
& +\left\|x_{t}-q\right\| \limsup _{n \rightarrow \infty}\left\|x_{n}-x_{t}\right\| \\
& +\rho\left\|q-x_{t}\right\| \limsup _{n \rightarrow \infty}\left\|x_{n}-x_{t}\right\| \\
& +\limsup _{n \rightarrow \infty}\left\langle f\left(x_{t}\right)-x_{t}, J\left(x_{n}-x_{t}\right)\right\rangle .
\end{aligned}
$$

Taking into account that $x_{t} \rightarrow q$ as $t \rightarrow 0$, we have from (82)

$$
\begin{aligned}
\limsup _{n \rightarrow \infty} & \left\langle f(q)-q, J\left(x_{n}-q\right)\right\rangle \\
\quad & =\limsup _{t \rightarrow 0} \limsup _{n \rightarrow \infty}\left\langle f(q)-q, J\left(x_{n}-q\right)\right\rangle \\
\quad \leq & \limsup \limsup _{t \rightarrow 0}\left\langle f(q)-q, J\left(x_{n}-q\right)-J\left(x_{n}-x_{t}\right)\right\rangle .
\end{aligned}
$$

Since $X$ has a uniformly Fréchet differentiable norm, the duality mapping $J$ is norm-to-norm uniformly continuous on bounded subsets of $X$. Consequently, the two limits are interchangeable, and hence (75) holds. From (68), we get $\left(y_{n}-q\right)-\left(x_{n}-q\right) \rightarrow 0$. Noticing that $J$ is norm-to-norm uniformly continuous on bounded subsets of $X$, we deduce from (75) that

$$
\begin{aligned}
& \limsup _{n \rightarrow \infty}\left\langle f(q)-q, J\left(y_{n}-q\right)\right\rangle \\
&=\limsup _{n \rightarrow \infty}\left(\left\langle f(q)-q, J\left(x_{n}-q\right)\right\rangle\right.\left.\quad+\left\langle f(q)-q, J\left(y_{n}-q\right)-J\left(x_{n}-q\right)\right\rangle\right) \\
& \\
&=\limsup _{n \rightarrow \infty}\left\langle f(q)-q, J\left(x_{n}-q\right)\right\rangle \leq 0 .
\end{aligned}
$$



that

Finally, let us show that $x_{n} \rightarrow q$ as $n \rightarrow \infty$. We observe

$$
\begin{aligned}
\left\|y_{n}-q\right\|^{2} & \\
=\| & \alpha_{n}\left(f\left(y_{n}\right)-f(q)\right)+\left(1-\alpha_{n}\right) \\
& \times\left(S_{n} G\left(x_{n}\right)-q\right)+\alpha_{n}(f(q)-q) \|^{2} \\
\leq & \| \alpha_{n}\left(f\left(y_{n}\right)-f(q)\right) \\
& +\left(1-\alpha_{n}\right)\left(S_{n} G\left(x_{n}\right)-q\right) \|^{2} \\
& +2 \alpha_{n}\left\langle f(q)-q, J\left(y_{n}-q\right)\right\rangle \\
\leq & \alpha_{n}\left\|f\left(y_{n}\right)-f(q)\right\|^{2} \\
& +\left(1-\alpha_{n}\right)\left\|S_{n} G\left(x_{n}\right)-q\right\|^{2} \\
& +2 \alpha_{n}\left\langle f(q)-q, J\left(y_{n}-q\right)\right\rangle \\
\leq & \alpha_{n} \rho\left\|y_{n}-q\right\|^{2}+\left(1-\alpha_{n}\right)\left\|x_{n}-q\right\|^{2} \\
& +2 \alpha_{n}\left\langle f(q)-q, J\left(y_{n}-q\right)\right\rangle,
\end{aligned}
$$

which implies that

$$
\begin{aligned}
\left\|y_{n}-q\right\|^{2} \leq & \left(1-\frac{1-\rho}{1-\alpha_{n} \rho} \alpha_{n}\right)\left\|x_{n}-q\right\|^{2} \\
& +\frac{\alpha_{n}(1-\rho)}{1-\alpha_{n} \rho} \cdot \frac{2\left\langle f(q)-q, J\left(y_{n}-q\right)\right\rangle}{1-\rho} .
\end{aligned}
$$

By (27) and the convexity of $\|\cdot\|^{2}$, we get

$$
\begin{aligned}
\| x_{n+1} & -q \|^{2} \\
& \leq \beta_{n}\left\|x_{n}-q\right\|^{2}+\gamma_{n}\left\|y_{n}-q\right\|^{2}+\delta_{n}\left\|S_{n} G\left(y_{n}\right)-q\right\|^{2} \\
& \leq \beta_{n}\left\|x_{n}-q\right\|^{2}+\gamma_{n}\left\|y_{n}-q\right\|^{2}+\delta_{n}\left\|y_{n}-q\right\|^{2} \\
& =\beta_{n}\left\|x_{n}-q\right\|^{2}+\left(1-\beta_{n}\right)\left\|y_{n}-q\right\|^{2},
\end{aligned}
$$

which together with (88) leads to

$$
\begin{aligned}
\| x_{n+1}- & q \|^{2} \\
\leq & \beta_{n}\left\|x_{n}-q\right\|^{2}+\left(1-\beta_{n}\right) \\
& \times\left\{\left(1-\frac{1-\rho}{1-\alpha_{n} \rho} \alpha_{n}\right)\left\|x_{n}-q\right\|^{2}\right. \\
& \left.+\frac{\alpha_{n}(1-\rho)}{1-\alpha_{n} \rho} \cdot \frac{2\left\langle f(q)-q, J\left(y_{n}-q\right)\right\rangle}{1-\rho}\right\} \\
= & {\left[1-\frac{\left(1-\beta_{n}\right)(1-\rho)}{1-\alpha_{n} \rho} \alpha_{n}\right]\left\|x_{n}-q\right\|^{2} } \\
& +\frac{\left(1-\beta_{n}\right)(1-\rho)}{1-\alpha_{n} \rho} \alpha_{n} \cdot \frac{2\left\langle f(q)-q, J\left(y_{n}-q\right)\right\rangle}{1-\rho} .
\end{aligned}
$$

Applying Lemma 2 to (88), we obtain that $x_{n} \rightarrow q$ as $n \rightarrow$ $\infty$. This completes the proof.

Corollary 15. Let $C$ be a nonempty, closed, and convex subset of a uniformly convex and 2-uniformly smooth Banach space $X$. Let $\Pi_{C}$ be a sunny nonexpansive retraction from $X$ onto $C$. Let the mapping $B_{i}: C \rightarrow X$ be $\alpha_{i}$-inverse-strongly accretive for $i=1,2$. Let $f: C \rightarrow C$ be a contraction with coefficient $\rho \in(0,1)$. Let $S$ be a nonexpansive mapping of $C$ into itself such that $F=\operatorname{Fix}(S) \cap \Omega \neq \emptyset$, where $\Omega$ is the fixed point set of the mapping $G=\Pi_{C}\left(I-\mu_{1} B_{1}\right) \Pi_{C}\left(I-\mu_{2} B_{2}\right)$. For arbitrarily given $x_{0} \in C$, let $\left\{x_{n}\right\}$ be the sequence generated by

$$
\begin{gathered}
y_{n}=\alpha_{n} f\left(y_{n}\right)+\left(1-\alpha_{n}\right) S G\left(x_{n}\right), \\
x_{n+1}=\beta_{n} x_{n}+\gamma_{n} y_{n}+\delta_{n} S G\left(y_{n}\right), \quad \forall n \geq 0,
\end{gathered}
$$

where $0<\mu_{i}<\alpha_{i} / \kappa^{2}$ for $i=1,2$ and $\left\{\alpha_{n}\right\},\left\{\beta_{n}\right\},\left\{\gamma_{n}\right\}$, and $\left\{\delta_{n}\right\}$ are the sequences in $(0,1)$ such that $\beta_{n}+\gamma_{n}+\delta_{n}=1, \forall n \geq 0$. Suppose that the following conditions hold:

(i) $\lim _{n \rightarrow \infty} \alpha_{n}=0$ and $\sum_{n=0}^{\infty} \alpha_{n}=\infty$,

(ii) $0<\liminf _{n \rightarrow \infty} \beta_{n} \leq \lim \sup _{n \rightarrow \infty}\left(\beta_{n}+\gamma_{n}\right)<1$ and $\liminf _{n \rightarrow \infty} \gamma_{n}>0$,

(iii) $\lim _{n \rightarrow \infty}\left|\left(\gamma_{n} /\left(1-\beta_{n}\right)\right)-\left(\gamma_{n-1} /\left(1-\beta_{n-1}\right)\right)\right|=0$.

Then $\left\{x_{n}\right\}$ converges strongly to $q \in F$, which solves the following VIP:

$$
\langle q-f(q), J(q-p)\rangle \leq 0, \quad \forall p \in F .
$$

Further, we illustrate Theorem 14 by virtue of an example, that is, the following corollary.

Corollary 16. Let $C$ be a nonempty, closed, and convex subset of a uniformly convex and 2-uniformly smooth Banach space $X$. Let $\Pi_{C}$ be a sunny nonexpansive retraction from $X$ onto $C$. Let $f: C \rightarrow C$ be a contraction with coefficient $\rho \in(0,1)$. Let $T$ be an $\eta$-strictly pseudocontractive mapping of $C$ into itself, and let $S$ be a nonexpansive mapping of $C$ into itself such that $F=\operatorname{Fix}(S) \cap \operatorname{Fix}(T) \neq \emptyset$. For arbitrarily given $x_{0} \in C$, let $\left\{x_{n}\right\}$ be the sequence generated by

$$
\begin{gathered}
y_{n}=\alpha_{n} f\left(y_{n}\right)+\left(1-\alpha_{n}\right) S(I-\lambda(I-T)) x_{n}, \\
x_{n+1}=\beta_{n} x_{n}+\gamma_{n} y_{n}+\delta_{n} S(I-\lambda(I-T)) y_{n}, \quad \forall n \geq 0,
\end{gathered}
$$

where $0<\lambda<\max \left\{1, \eta / \kappa^{2}\right\}$ and $\left\{\alpha_{n}\right\},\left\{\beta_{n}\right\},\left\{\gamma_{n}\right\}$, and $\left\{\delta_{n}\right\}$ are the sequences in $(0,1)$ such that $\beta_{n}+\gamma_{n}+\delta_{n}=1, \forall n \geq 0$. Suppose that the following conditions hold:

(i) $\lim _{n \rightarrow \infty} \alpha_{n}=0$ and $\sum_{n=0}^{\infty} \alpha_{n}=\infty$,

(ii) $0<\liminf _{n \rightarrow \infty} \beta_{n} \leq \limsup _{n \rightarrow \infty}\left(\beta_{n}+\gamma_{n}\right)<1$ and $\liminf _{n \rightarrow \infty} \gamma_{n}>0$,

(iii) $\lim _{n \rightarrow \infty}\left|\left(\gamma_{n} /\left(1-\beta_{n}\right)\right)-\left(\gamma_{n-1} /\left(1-\beta_{n-1}\right)\right)\right|=0$.

Then $\left\{x_{n}\right\}$ converges strongly to $q \in F$, which solves the following VIP:

$$
\langle q-f(q), J(q-p)\rangle \leq 0, \quad \forall p \in F .
$$


Proof. In Corollary 15, put $B_{1}=I-T, B_{2}=0, \mu_{1}=\lambda$, and $\alpha_{1}=\eta$. Since $T$ is an $\eta$-strictly pseudocontractive mapping, it is clear that $B_{1}=I-T$ is an $\eta$-inverse strongly accretive mapping. Hence, the GSVI (9) is equivalent to the following VIP of finding $x^{*} \in C$ such that

$$
\left(B_{1} x^{*}, J\left(x-x^{*}\right)\right) \geq 0, \quad \forall x \in C,
$$

which leads to $\Omega=\operatorname{VI}\left(C, B_{1}\right)$. In the meantime, we have

$$
\begin{aligned}
G x_{n} & =\Pi_{C}\left(I-\mu_{1} B_{1}\right) \Pi_{C}\left(I-\mu_{2} B_{2}\right) x_{n} \\
& =\Pi_{C}\left(I-\mu_{1} B_{1}\right) x_{n} \\
& =\Pi_{C}\left[(1-\lambda) x_{n}+\lambda T x_{n}\right] \\
& =x_{n}-\lambda(I-T) x_{n} .
\end{aligned}
$$

In the same way, we get $G y_{n}=y_{n}-\lambda(I-T) y_{n}$. In this case, it is easy to see that (91) reduces to (93). We claim that $\operatorname{Fix}(T)=$ $\operatorname{VI}\left(C, B_{1}\right)$. As a matter of fact, we have, for $\lambda>0$,

$$
\begin{aligned}
u \in \mathrm{VI} & \left(C, B_{1}\right) \\
& \Longleftrightarrow\left\langle B_{1} u, J(y-u)\right\rangle \geq 0 \quad \forall y \in C \\
& \Longleftrightarrow\left\langle u-\lambda B_{1} u-u, J(u-y)\right\rangle \geq 0 \quad \forall y \in C \\
& \Longleftrightarrow u=\Pi_{C}\left(u-\lambda B_{1} u\right) \\
& \Longleftrightarrow u=\Pi_{C}(u-\lambda u+\lambda T u) \\
& \Longleftrightarrow\langle u-\lambda u+\lambda T u-u, J(u-y)\rangle \geq 0 \quad \forall y \in C \\
& \Longleftrightarrow\langle u-T u, J(u-y)\rangle \leq 0 \quad \forall y \in C \quad \\
& \Longleftrightarrow u=T u \\
& \Longleftrightarrow u \in \operatorname{Fix}(T) .
\end{aligned}
$$

So, we conclude that $F=\operatorname{Fix}(S) \cap \Omega=\operatorname{Fix}(S) \cap \operatorname{Fix}(T)$. Therefore, the desired result follows from Corollary 15.

Remark 17. Theorem 14 improves, extends, supplements, and develops Cai and Bu [2, Theorem 3.1 and Corollary 3.2] and Jung [5, Theorem 3.1] in the following aspects.

(i) The problem of finding a point $q \in \bigcap_{n} \operatorname{Fix}\left(S_{n}\right) \cap \Omega$ in Theorem 14 is more general and more subtle than the problem of finding a point $q \in \operatorname{Fix}(S) \cap \operatorname{VI}(C, A)$ in Jung [5, Theorem 3.1].

(ii) The iterative scheme in [2, Theorem 3.1] is extended to develop the iterative scheme (27) of Theorem 14 by virtue of the iterative scheme of [5, Theorem 3.1]. The iterative scheme (27) of Theorem 14 is more advantageous and more flexible than the iterative scheme of [2, Theorem 3.1] because it involves several parameter sequences $\left\{\alpha_{n}\right\},\left\{\beta_{n}\right\},\left\{\gamma_{n}\right\}$, and $\left\{\delta_{n}\right\}$.

(iii) The iterative scheme (27) in Theorem 14 is very different from everyone in both [2, Theorem 3.1] and [5, Theorem 3.1] because the mappings $S_{n}$ and $G$ in the iterative scheme of [2, Theorem 3.1] and the mapping $S P_{C}\left(I-\lambda_{n} A\right)$ in the iterative scheme of [5, Theorem 3.1] are replaced by the same composite mapping $S_{n} G$ in the iterative scheme (27) of Theorem 14.

(iv) The proof in [2, Theorem 3.1] depends on the argument techniques in [3], the inequality in 2-uniformly smooth Banach spaces ([9]), and the inequality in smooth and uniform convex Banach spaces ([14, Proposition 1]). Because the composite mapping $S_{n} G$ appears in the iterative scheme (27) of Theorem 14, the proof of Theorem 14 depends on the argument techniques in [3], the inequality in 2-uniformly smooth Banach spaces, the inequality in smooth and uniform convex Banach spaces, and the inequality in uniform convex Banach spaces (Lemma 6).

(v) The iterative scheme in [2, Corollary 3.2] is extended to develop the new iterative scheme in Corollary 15 because the mappings $S$ and $G$ are replaced by the same composite mapping $S G$ in Corollary 15.

\section{Explicit Iterative Schemes}

In this section, we introduce our explicit iterative schemes and show the strong convergence theorems. First, we give several useful lemmas.

Lemma 18. Let $C$ be a nonempty, closed, and convex subset of a smooth Banach space $X$, and let the mapping $B_{i}: C \rightarrow X$ be $\lambda_{i}$-strictly pseudocontractive and $\alpha_{i}$-strongly accretive with $\alpha_{i}+\lambda_{i} \geq 1$ for $i=1,2$. Then, for $\mu_{i} \in(0,1]$, we have

$$
\begin{array}{r}
\left\|\left(I-\mu_{i} B_{i}\right) x-\left(I-\mu_{i} B_{i}\right) y\right\| \\
\leq\left\{\sqrt{\frac{1-\alpha_{i}}{\lambda_{i}}}+\left(1-\mu_{i}\right)\left(1+\frac{1}{\lambda_{i}}\right)\right\}\|x-y\|, \\
\forall x, y \in C,
\end{array}
$$

for $i=1$, 2. In particular, if $1-\left(\lambda_{i} /\left(1+\lambda_{i}\right)\right)\left(1-\sqrt{\left(1-\alpha_{i}\right) / \lambda_{i}}\right) \leq$ $\mu_{i} \leq 1$, then $I-\mu_{i} B_{i}$ is nonexpansive for $i=1,2$.

Proof. Taking into account the $\lambda_{i}$-strict pseudocontractivity of $B_{i}$, we derive for every $x, y \in C$

$$
\begin{aligned}
\lambda_{i} \|(I & \left.-B_{i}\right) x-\left(I-B_{i}\right) y \|^{2} \\
& \leq\left\langle\left(I-B_{i}\right) x-\left(I-B_{i}\right) y, J(x-y)\right\rangle \\
& \leq\left\|\left(I-B_{i}\right) x-\left(I-B_{i}\right) y\right\|\|x-y\|,
\end{aligned}
$$

which implies that

$$
\left\|\left(I-B_{i}\right) x-\left(I-B_{i}\right) y\right\| \leq \frac{1}{\lambda_{i}}\|x-y\| .
$$


Hence,

$$
\begin{aligned}
& \left\|B_{i} x-B_{i} y\right\| \\
& \quad \leq\left\|\left(I-B_{i}\right) x-\left(I-B_{i}\right) y\right\|+\|x-y\| \\
& \quad \leq\left(1+\frac{1}{\lambda_{i}}\right)\|x-y\| .
\end{aligned}
$$

Utilizing the $\alpha_{i}$-strong accretivity and $\lambda_{i}$-strict pseudocontractivity of $B_{i}$, we get

$$
\begin{aligned}
\lambda_{i} \|(I & \left.-B_{i}\right) x-\left(I-B_{i}\right) y \|^{2} \\
& \leq\|x-y\|^{2}-\left\langle B_{i} x-B_{i} y, J(x-y)\right\rangle \\
& \leq\left(1-\alpha_{i}\right)\|x-y\|^{2} .
\end{aligned}
$$

So, we have

$$
\left\|\left(I-B_{i}\right) x-\left(I-B_{i}\right) y\right\| \leq \sqrt{\frac{1-\alpha_{i}}{\lambda_{i}}}\|x-y\| .
$$

Therefore, for $\mu_{i} \in(0,1]$, we have

$$
\begin{aligned}
\|(I- & \left.\mu_{i} B_{i}\right) x-\left(I-\mu_{i} B_{i}\right) y \| \\
& \leq\left\|\left(I-B_{i}\right) x-\left(I-B_{i}\right) y\right\|+\left(1-\mu_{i}\right)\left\|B_{i} x-B_{i} y\right\| \\
& \leq \sqrt{\frac{1-\alpha_{i}}{\lambda_{i}}}\|x-y\|+\left(1-\mu_{i}\right)\left(1+\frac{1}{\lambda_{i}}\right)\|x-y\| \\
& =\left\{\sqrt{\frac{1-\alpha_{i}}{\lambda_{i}}}+\left(1-\mu_{i}\right)\left(1+\frac{1}{\lambda_{i}}\right)\right\}\|x-y\| .
\end{aligned}
$$

Since $1-\left(\lambda_{i} /\left(1+\lambda_{i}\right)\right)\left(1-\sqrt{\left(1-\alpha_{\mathrm{i}}\right) / \lambda_{i}}\right) \leq \mu_{i} \leq 1$, it follows immediately that

$$
\sqrt{\frac{1-\alpha_{i}}{\lambda_{i}}}+\left(1-\mu_{i}\right)\left(1+\frac{1}{\lambda_{i}}\right) \leq 1
$$

This implies that $I-\mu_{i} B_{i}$ is nonexpansive for $i=1,2$.

Lemma 19. Let $C$ be a nonempty, closed, and convex subset of a smooth Banach space $X$. Let $\Pi_{C}$ be a sunny nonexpansive retraction from $X$ onto $C$, and let the mapping $B_{i}: C \rightarrow X$ be $\lambda_{i}$-strictly pseudocontractive and $\alpha_{i}$-strongly accretive with $\alpha_{i}+\lambda_{i} \geq 1$ for $i=1,2$. Let $G: C \rightarrow C$ be the mapping defined by

$$
\begin{aligned}
G(x)=\Pi_{C} & {\left[\Pi_{C}\left(x-\mu_{2} B_{2} x\right)\right.} \\
& \left.-\mu_{1} B_{1} \Pi_{C}\left(x-\mu_{2} B_{2} x\right)\right], \quad \forall x \in C .
\end{aligned}
$$

If $1-\left(\lambda_{i} /\left(1+\lambda_{i}\right)\right)\left(1-\sqrt{\left(1-\alpha_{i}\right) / \lambda_{i}}\right) \leq \mu_{i} \leq 1$, then $G: C \rightarrow C$ is nonexpansive.
Proof. According to Lemma 10, we know that $I-\mu_{i} B_{i}$ is nonexpansive for $i=1,2$. Hence, for all $x, y \in C$, we have

$$
\begin{aligned}
\|G(x)-G(y)\| \\
=\| \Pi_{C}\left[\Pi_{C}\left(x-\mu_{2} B_{2} x\right)-\mu_{1} B_{1} \Pi_{C}\left(x-\mu_{2} B_{2} x\right)\right] \\
\quad \quad-\Pi_{C}\left[\Pi_{C}\left(y-\mu_{2} B_{2} y\right)-\mu_{1} B_{1} \Pi_{C}\left(y-\mu_{2} B_{2} y\right)\right] \| \\
=\| \Pi_{C}\left(I-\mu_{1} B_{1}\right) \Pi_{C}\left(I-\mu_{2} B_{2}\right) x \\
\quad \quad-\Pi_{C}\left(I-\mu_{1} B_{1}\right) \Pi_{C}\left(I-\mu_{2} B_{2}\right) y \| \\
\leq \|\left(I-\mu_{1} B_{1}\right) \Pi_{C}\left(I-\mu_{2} B_{2}\right) x \\
\quad-\left(I-\mu_{1} B_{1}\right) \Pi_{C}\left(I-\mu_{2} B_{2}\right) y \| \\
\leq\left\|\Pi_{C}\left(I-\mu_{2} B_{2}\right) x-\Pi_{C}\left(I-\mu_{2} B_{2}\right) y\right\| \\
\leq\left\|\left(I-\mu_{2} B_{2}\right) x-\left(I-\mu_{2} B_{2}\right) y\right\| \\
\leq\|x-y\| .
\end{aligned}
$$

This shows that $G: C \rightarrow C$ is nonexpansive. This completes the proof.

Lemma 20. Let $C$ be a nonempty, closed, and convex subset of a smooth Banach space $X$. Let $\Pi_{C}$ be a sunny nonexpansive retraction from $X$ onto $C$, and let the mapping $B_{i}: C \rightarrow X$ be $\lambda_{i}$-strictly pseudocontractive and $\alpha_{i}$-strongly accretive for $i=$ 1,2 . For given $x^{*}, y^{*} \in C,\left(x^{*}, y^{*}\right)$ is a solution of GSVI (9) if and only if $x^{*}=\Pi_{C}\left(y^{*}-\mu_{1} B_{1} y^{*}\right)$ where $y^{*}=\Pi_{C}\left(x^{*}-\right.$ $\left.\mu_{2} B_{2} x^{*}\right)$.

Proof. We can rewrite GSVI (9) as

$$
\begin{aligned}
& \left\langle x^{*}-\left(y^{*}-\mu_{1} B_{1} y^{*}\right), J\left(x-x^{*}\right)\right\rangle \geq 0, \quad \forall x \in C, \\
& \left\langle y^{*}-\left(x^{*}-\mu_{2} B_{2} x^{*}\right), J\left(x-y^{*}\right)\right\rangle \geq 0, \quad \forall x \in C,
\end{aligned}
$$

which is obviously equivalent to

$$
\begin{aligned}
& x^{*}=\Pi_{C}\left(y^{*}-\mu_{1} B_{1} y^{*}\right), \\
& y^{*}=\Pi_{C}\left(x^{*}-\mu_{2} B_{2} x^{*}\right),
\end{aligned}
$$

because of Lemma 5. This completes the proof.

Remark 21. By Lemma 20, we observe that

$$
x^{*}=\Pi_{C}\left[\Pi_{C}\left(x^{*}-\mu_{2} B_{2} x^{*}\right)-\mu_{1} B_{1} \Pi_{C}\left(x^{*}-\mu_{2} B_{2} x^{*}\right)\right],
$$

which implies that $x^{*}$ is a fixed point of the mapping $G$. Throughout this paper, the set of fixed points of the mapping $G$ is denoted by $\Omega$.

We are now in a position to state and prove our result on the explicit iterative scheme.

Theorem 22. Let $C$ be a nonempty, closed, and convex subset of a uniformly convex Banach space $X$ which has a uniformly 
Gâteaux differentiable norm. Let $\Pi_{C}$ be a sunny nonexpansive retraction from $X$ onto $C$. Let the mapping $B_{i}: C \rightarrow X$ be $\lambda_{i^{-}}$ strictly pseudocontractive and $\alpha_{i}$-strongly accretive with $\alpha_{i}+$ $\lambda_{i} \geq 1$ for $i=1,2$. Let $f: C \rightarrow C$ be a contraction with coefficient $\rho \in(0,1)$. Let $\left\{S_{n}\right\}_{n=0}^{\infty}$ be an infinite family of nonexpansive mappings of $C$ into itself such that $F=$ $\cap_{n=0}^{\infty} \operatorname{Fix}\left(S_{n}\right) \cap \Omega \neq \emptyset$, where $\Omega$ is the fixed point set of the mapping $G=\Pi_{C}\left(I-\mu_{1} B_{1}\right) \Pi_{C}\left(I-\mu_{2} B_{2}\right)$. For arbitrarily given $x_{0} \in C$, let $\left\{x_{n}\right\}$ be the sequence generated by

$$
\begin{gathered}
y_{n}=\alpha_{n} G\left(x_{n}\right)+\left(1-\alpha_{n}\right) S_{n} G\left(x_{n}\right), \\
x_{n+1}=\beta_{n} f\left(x_{n}\right)+\gamma_{n} y_{n}+\delta_{n} S_{n} G\left(y_{n}\right), \quad \forall n \geq 0,
\end{gathered}
$$

where $1-\left(\lambda_{i} /\left(1+\lambda_{i}\right)\right)\left(1-\sqrt{\left(1-\alpha_{i}\right) / \lambda_{i}}\right) \leq \mu_{i} \leq 1$ for $i=1,2$ and $\left\{\alpha_{n}\right\},\left\{\beta_{n}\right\},\left\{\gamma_{n}\right\}$, and $\left\{\delta_{n}\right\}$ are the sequences in $(0,1)$ such that $\beta_{n}+\gamma_{n}+\delta_{n}=1, \forall n \geq 0$. Suppose that the following conditions hold:

(i) $0<\liminf _{n \rightarrow \infty} \alpha_{n} \leq \limsup _{n \rightarrow \infty} \alpha_{n}<1$,

(ii) $\lim _{n \rightarrow \infty} \beta_{n}=0$ and $\sum_{n=0}^{\infty} \beta_{n}=\infty$,

(iii) $\sum_{n=1}^{\infty}\left|\alpha_{n}-\alpha_{n-1}\right|<\infty$ or $\lim _{n \rightarrow \infty}\left|\alpha_{n}-\alpha_{n-1}\right| / \beta_{n}=0$,

(iv) $\sum_{n=1}^{\infty}\left|\beta_{n}-\beta_{n-1}\right|<\infty$ or $\lim _{n \rightarrow \infty} \beta_{n-1} / \beta_{n}=1$,

(v) $\sum_{n=1}^{\infty}\left|\left(\gamma_{n} /\left(1-\beta_{n}\right)\right)-\left(\gamma_{n-1} /\left(1-\beta_{n-1}\right)\right)\right|<\infty$ or $\lim _{n \rightarrow \infty}\left(1 / \beta_{n}\right)\left|\left(\gamma_{n} /\left(1-\beta_{n}\right)\right)-\left(\gamma_{n-1} /\left(1-\beta_{n-1}\right)\right)\right|=0$,

(vi) $0<\liminf _{n \rightarrow \infty} \gamma_{n} \leq \limsup _{n \rightarrow \infty} \gamma_{n}<1$.

Assume that $\sum_{n=1}^{\infty} \sup _{x \in D}\left\|S_{n} x-S_{n-1} x\right\|<\infty$ for any bounded subset $D$ of $C$, and let $S$ be a mapping of $C$ into itself defined by $S x=\lim _{n \rightarrow \infty} S_{n} x$ for all $x \in C$. Suppose that $\operatorname{Fix}(S)=$ $\bigcap_{n=0}^{\infty} \operatorname{Fix}\left(S_{n}\right)$. Then $\left\{x_{n}\right\}$ converges strongly to $q \in F$, which solves the following VIP:

$$
\langle q-f(q), J(q-p)\rangle \leq 0, \quad \forall p \in F .
$$

Proof. Take a fixed $p \in F$ arbitrarily. Then by Lemma 20, we know that $p=G(p)$ and $p=S_{n} p$ for all $n \geq 0$. Moreover, by Lemma 19, we have

$$
\begin{aligned}
& \left\|y_{n}-p\right\| \\
& \quad \leq \alpha_{n}\left\|G\left(x_{n}\right)-p\right\|+\left(1-\alpha_{n}\right)\left\|S_{n} G\left(x_{n}\right)-p\right\| \\
& \quad \leq \alpha_{n}\left\|x_{n}-p\right\|+\left(1-\alpha_{n}\right)\left\|x_{n}-p\right\| \\
& \quad=\left\|x_{n}-p\right\| .
\end{aligned}
$$

From (113) we obtain

$$
\begin{aligned}
\| x_{n+1}- & p \| \\
\leq & \beta_{n}\left\|f\left(x_{n}\right)-p\right\|+\gamma_{n}\left\|y_{n}-p\right\| \\
& +\delta_{n}\left\|S_{n} G\left(y_{n}\right)-p\right\| \\
\leq & \beta_{n}\left(\left\|f\left(x_{n}\right)-f(p)\right\|+\|f(p)-p\|\right) \\
& +\gamma_{n}\left\|y_{n}-p\right\|+\delta_{n}\left\|y_{n}-p\right\| \\
\leq & \beta_{n} \rho\left\|x_{n}-p\right\|+\beta_{n}\|f(p)-p\| \\
& +\left(1-\beta_{n}\right)\left\|y_{n}-p\right\| \\
\leq & \beta_{n} \rho\left\|x_{n}-p\right\|+\beta_{n}\|f(p)-p\| \\
& +\left(1-\beta_{n}\right)\left\|x_{n}-p\right\| \\
= & \left(1-\beta_{n}(1-\rho)\right)\left\|x_{n}-p\right\| \\
& +\beta_{n}\|f(p)-p\| \\
= & \left(1-\beta_{n}(1-\rho)\right)\left\|x_{n}-p\right\| \\
& +\beta_{n}(1-\rho) \cdot \frac{\|f(p)-p\|}{1-\rho} \\
\leq & \max \left\{\left\|x_{0}-p\right\|, \frac{\|f(p)-p\|}{1-\rho}\right\}, \\
\quad & (1-p) \\
& (1-p) \\
& \\
&
\end{aligned}
$$

which implies that $\left\{x_{n}\right\}$ is bounded. By Lemma 19 we know from (113) that $\left\{y_{n}\right\},\left\{G\left(x_{n}\right)\right\}$, and $\left\{G\left(y_{n}\right)\right\}$ are bounded.

Let us show that $\left\|x_{n+1}-x_{n}\right\| \rightarrow 0$ and $\left\|x_{n}-y_{n}\right\| \rightarrow 0$ as $n \rightarrow \infty$. As a matter of fact, from (113), we have

$$
\begin{gathered}
y_{n}=\alpha_{n} G\left(x_{n}\right)+\left(1-\alpha_{n}\right) S_{n} G\left(x_{n}\right), \\
y_{n-1}=\alpha_{n-1} G\left(x_{n-1}\right)+\left(1-\alpha_{n-1}\right) S_{n-1} G\left(x_{n-1}\right), \quad \forall n \geq 1 .
\end{gathered}
$$

Simple calculations show that

$$
\begin{aligned}
y_{n}-y_{n-1}= & \alpha_{n}\left(G\left(x_{n}\right)-G\left(x_{n-1}\right)\right) \\
& +\left(\alpha_{n}-\alpha_{n-1}\right)\left(G\left(x_{n-1}\right)-S_{n-1} G\left(x_{n-1}\right)\right) \\
& +\left(1-\alpha_{n}\right)\left(S_{n} G\left(x_{n}\right)-S_{n-1} G\left(x_{n-1}\right)\right) .
\end{aligned}
$$

It follows that

$$
\begin{aligned}
\| y_{n}- & y_{n-1} \| \\
\leq & \alpha_{n}\left\|G\left(x_{n}\right)-G\left(x_{n-1}\right)\right\| \\
& +\left|\alpha_{n}-\alpha_{n-1}\right|\left\|G\left(x_{n-1}\right)-S_{n-1} G\left(x_{n-1}\right)\right\| \\
& +\left(1-\alpha_{n}\right)\left\|S_{n} G\left(x_{n}\right)-S_{n-1} G\left(x_{n-1}\right)\right\|
\end{aligned}
$$




$$
\begin{aligned}
\leq & \alpha_{n}\left\|G\left(x_{n}\right)-G\left(x_{n-1}\right)\right\| \\
& +\left|\alpha_{n}-\alpha_{n-1}\right|\left\|G\left(x_{n-1}\right)-S_{n-1} G\left(x_{n-1}\right)\right\| \\
& +\left(1-\alpha_{n}\right)\left(\left\|S_{n} G\left(x_{n}\right)-S_{n} G\left(x_{n-1}\right)\right\|\right. \\
& \left.\quad+\left\|S_{n} G\left(x_{n-1}\right)-S_{n-1} G\left(x_{n-1}\right)\right\|\right) \\
\leq & \alpha_{n}\left\|G\left(x_{n}\right)-G\left(x_{n-1}\right)\right\| \\
& +\left|\alpha_{n}-\alpha_{n-1}\right|\left\|G\left(x_{n-1}\right)-S_{n-1} G\left(x_{n-1}\right)\right\| \\
& +\left(1-\alpha_{n}\right)\left(\left\|G\left(x_{n}\right)-G\left(x_{n-1}\right)\right\|\right. \\
& \left.\quad+\left\|S_{n} G\left(x_{n-1}\right)-S_{n-1} G\left(x_{n-1}\right)\right\|\right) \\
\leq & \left\|G\left(x_{n}\right)-G\left(x_{n-1}\right)\right\| \\
& +\left|\alpha_{n}-\alpha_{n-1}\right|\left\|G\left(x_{n-1}\right)-S_{n-1} G\left(x_{n-1}\right)\right\| \\
& +\left\|S_{n} G\left(x_{n-1}\right)-S_{n-1} G\left(x_{n-1}\right)\right\| \\
\leq & \left\|x_{n}-x_{n-1}\right\|+\left|\alpha_{n}-\alpha_{n-1}\right| \\
& \times\left\|G\left(x_{n-1}\right)-S_{n-1} G\left(x_{n-1}\right)\right\| \\
& +\left\|S_{n} G\left(x_{n-1}\right)-S_{n-1} G\left(x_{n-1}\right)\right\| .
\end{aligned}
$$

Now, we write $x_{n}=\beta_{n-1} f\left(x_{n-1}\right)+\left(1-\beta_{n-1}\right) v_{n-1}, \forall n \geq 1$, where $v_{n-1}=\left(x_{n}-\beta_{n-1} f\left(x_{n-1}\right)\right) /\left(1-\beta_{n-1}\right)$. It follows that, for all $n \geq 1$,

$$
\begin{aligned}
v_{n}-v_{n-1} & \\
= & \frac{x_{n+1}-\beta_{n} f\left(x_{n}\right)}{1-\beta_{n}}-\frac{x_{n}-\beta_{n-1} f\left(x_{n-1}\right)}{1-\beta_{n-1}} \\
= & \frac{\gamma_{n} y_{n}+\delta_{n} S_{n} G\left(y_{n}\right)}{1-\beta_{n}} \\
& -\frac{\gamma_{n-1} y_{n-1}+\delta_{n-1} S_{n-1} G\left(y_{n-1}\right)}{1-\beta_{n-1}} \\
= & \frac{\gamma_{n}\left(y_{n}-y_{n-1}\right)+\delta_{n}\left(S_{n} G\left(y_{n}\right)-S_{n-1} G\left(y_{n-1}\right)\right)}{1-\beta_{n}} \\
& +\left(\frac{\gamma_{n}}{1-\beta_{n}}-\frac{\gamma_{n-1}}{1-\beta_{n-1}}\right) y_{n-1} \\
& +\left(\frac{\delta_{n}}{1-\beta_{n}}-\frac{\delta_{n-1}}{1-\beta_{n-1}}\right) S_{n-1} G\left(y_{n-1}\right) .
\end{aligned}
$$

This together with (117) implies that

$$
\begin{aligned}
\| v_{n}- & v_{n-1} \| \\
\leq & \frac{\left\|\gamma_{n}\left(y_{n}-y_{n-1}\right)+\delta_{n}\left(S_{n} G\left(y_{n}\right)-S_{n-1} G\left(y_{n-1}\right)\right)\right\|}{1-\beta_{n}} \\
& +\left|\frac{\gamma_{n}}{1-\beta_{n}}-\frac{\gamma_{n-1}}{1-\beta_{n-1}}\right|\left\|y_{n-1}\right\| \\
& +\left|\frac{\delta_{n}}{1-\beta_{n}}-\frac{\delta_{n-1}}{1-\beta_{n-1}}\right|\left\|S_{n-1} G\left(y_{n-1}\right)\right\|
\end{aligned}
$$

$$
\begin{aligned}
\leq & \left(\gamma_{n}\left\|y_{n}-y_{n-1}\right\|\right. \\
& +\delta_{n}\left(\left\|S_{n} G\left(y_{n}\right)-S_{n} G\left(y_{n-1}\right)\right\|\right. \\
& \left.\left.\quad+\left\|S_{n} G\left(y_{n-1}\right)-S_{n-1} G\left(y_{n-1}\right)\right\|\right)\right) \\
& +\left|\frac{\gamma_{n}}{1-\beta_{n}}-\frac{\gamma_{n-1}}{1-\beta_{n-1}}\right|\left\|y_{n-1}\right\| \\
& +\left|\frac{\gamma_{n}}{1-\beta_{n}}-\frac{\gamma_{n-1}}{1-\beta_{n-1}}\right|\left\|S_{n-1} G\left(y_{n-1}\right)\right\| \\
\leq & \frac{\gamma_{n}\left\|y_{n}-y_{n-1}\right\|+\delta_{n}\left\|y_{n}-y_{n-1}\right\|}{\gamma_{n}+\delta_{n}} \\
& +\frac{\delta_{n}}{\gamma_{n}+\delta_{n}}\left\|S_{n} G\left(y_{n-1}\right)-S_{n-1} G\left(y_{n-1}\right)\right\| \\
& +\left|\frac{\gamma_{n}}{1-\beta_{n}}-\frac{\gamma_{n-1}}{1-\beta_{n-1}}\right| \\
& \times\left(\left\|y_{n-1}\right\|+\left\|S_{n-1} G\left(y_{n-1}\right)\right\|\right) \\
\leq & \left\|y_{n}-y_{n-1}\right\|+\left\|S_{n} G\left(y_{n-1}\right)-S_{n-1} G\left(y_{n-1}\right)\right\| \\
& +\left|\frac{\gamma_{n}}{1-\beta_{n}}-\frac{\gamma_{n-1}}{1-\beta_{n-1}}\right|\left(\left\|y_{n-1}\right\|+\left\|S_{n-1} G\left(y_{n-1}\right)\right\|\right) \\
\leq & \left\|x_{n}-x_{n-1}\right\|+\left|\alpha_{n}-\alpha_{n-1}\right|\left\|G\left(x_{n-1}\right)-S_{n-1} G\left(x_{n-1}\right)\right\| \\
+ & +\left|\frac{\gamma_{n} G\left(x_{n-1}\right)-S_{n-1} G\left(x_{n-1}\right) \|}{1-\beta_{n}}-\frac{\gamma_{n-1}}{1-\beta_{n-1}}\right|\left(\left\|y_{n-1}\right\|+\left\|S_{n-1} G\left(y_{n-1}\right)\right\|\right) . \\
+ & \mid S_{n} G\left(y_{n-1}\right)-S_{n-1} G\left(y_{n-1}\right) \| \\
&
\end{aligned}
$$

Furthermore, we note that

$$
\begin{gathered}
x_{n+1}=\beta_{n} f\left(x_{n}\right)+\left(1-\beta_{n}\right) v_{n}, \\
x_{n}=\beta_{n-1} f\left(x_{n-1}\right)+\left(1-\beta_{n-1}\right) v_{n-1}, \quad \forall n \geq 1 .
\end{gathered}
$$

Also, simple calculations show that

$$
\begin{aligned}
x_{n+1}-x_{n}= & \beta_{n}\left(f\left(x_{n}\right)-f\left(x_{n-1}\right)\right)+\left(\beta_{n}-\beta_{n-1}\right) \\
& \times\left(f\left(x_{n-1}\right)-v_{n-1}\right)+\left(1-\beta_{n}\right)\left(v_{n}-v_{n-1}\right) .
\end{aligned}
$$

This together with (119) implies that

$$
\begin{aligned}
\| x_{n+1}- & x_{n} \| \\
\leq & \beta_{n}\left\|f\left(x_{n}\right)-f\left(x_{n-1}\right)\right\|+\left|\beta_{n}-\beta_{n-1}\right| \\
& \quad \times\left\|f\left(x_{n-1}\right)-v_{n-1}\right\|+\left(1-\beta_{n}\right)\left\|v_{n}-v_{n-1}\right\|
\end{aligned}
$$




$$
\begin{aligned}
& \leq \beta_{n} \rho\left\|x_{n}-x_{n-1}\right\| \\
& +\left|\beta_{n}-\beta_{n-1}\right|\left\|f\left(x_{n-1}\right)-v_{n-1}\right\|+\left(1-\beta_{n}\right) \\
& \times\left[\left\|x_{n}-x_{n-1}\right\|\right. \\
& +\left|\alpha_{n}-\alpha_{n-1}\right|\left\|G\left(x_{n-1}\right)-S_{n-1} G\left(x_{n-1}\right)\right\| \\
& +\left\|S_{n} G\left(x_{n-1}\right)-S_{n-1} G\left(x_{n-1}\right)\right\| \\
& +\left\|S_{n} G\left(y_{n-1}\right)-S_{n-1} G\left(y_{n-1}\right)\right\| \\
& +\left|\frac{\gamma_{n}}{1-\beta_{n}}-\frac{\gamma_{n-1}}{1-\beta_{n-1}}\right| \\
& \left.\times\left(\left\|y_{n-1}\right\|+\left\|S_{n-1} G\left(y_{n-1}\right)\right\|\right)\right] \\
& \leq\left(1-\beta_{n}(1-\rho)\right)\left\|x_{n}-x_{n-1}\right\| \\
& +\left|\beta_{n}-\beta_{n-1}\right|\left\|f\left(x_{n-1}\right)-v_{n-1}\right\| \\
& +\left|\alpha_{n}-\alpha_{n-1}\right|\left\|G\left(x_{n-1}\right)-S_{n-1} G\left(x_{n-1}\right)\right\| \\
& +\left\|S_{n} G\left(x_{n-1}\right)-S_{n-1} G\left(x_{n-1}\right)\right\| \\
& +\left\|S_{n} G\left(y_{n-1}\right)-S_{n-1} G\left(y_{n-1}\right)\right\| \\
& +\left|\frac{\gamma_{n}}{1-\beta_{n}}-\frac{\gamma_{n-1}}{1-\beta_{n-1}}\right|\left(\left\|y_{n-1}\right\|+\left\|S_{n-1} G\left(y_{n-1}\right)\right\|\right) \\
& \leq\left(1-\beta_{n}(1-\rho)\right)\left\|x_{n}-x_{n-1}\right\| \\
& +\left|\beta_{n}-\beta_{n-1}\right| M+\left|\alpha_{n}-\alpha_{n-1}\right| M \\
& +\left\|S_{n} G\left(x_{n-1}\right)-S_{n-1} G\left(x_{n-1}\right)\right\| \\
& +\left\|S_{n} G\left(y_{n-1}\right)-S_{n-1} G\left(y_{n-1}\right)\right\| \\
& +\left|\frac{\gamma_{n}}{1-\beta_{n}}-\frac{\gamma_{n-1}}{1-\beta_{n-1}}\right| M \\
& =\left(1-\beta_{n}(1-\rho)\right)\left\|x_{n}-x_{n-1}\right\| \\
& +M\left(\left|\alpha_{n}-\alpha_{n-1}\right|\right. \\
& \left.+\left|\beta_{n}-\beta_{n-1}\right|+\left|\frac{\gamma_{n}}{1-\beta_{n}}-\frac{\gamma_{n-1}}{1-\beta_{n-1}}\right|\right) \\
& +\left\|S_{n} G\left(x_{n-1}\right)-S_{n-1} G\left(x_{n-1}\right)\right\| \\
& +\left\|S_{n} G\left(y_{n-1}\right)-S_{n-1} G\left(y_{n-1}\right)\right\| \text {, }
\end{aligned}
$$

where $\sup _{n \geq 0}\left\{\left\|f\left(x_{n}\right)\right\|+\left\|v_{n}\right\|+\left\|G\left(x_{n}\right)\right\|+\left\|S_{n} G\left(x_{n}\right)\right\|+\right.$ $\left.\left\|y_{n}\right\|+\left\|S_{n} G\left(y_{n}\right)\right\|\right\} \leq M$ for some $M>0$. Utilizing Lemma 2, from conditions (ii)-(v) and the assumption on $\left\{S_{n}\right\}$, we deduce that

$$
\lim _{n \rightarrow \infty}\left\|x_{n+1}-x_{n}\right\|=0 .
$$

Taking into account the boundedness of $\left\{G\left(x_{n}\right)\right\}$ and $\left\{S_{n} G\left(x_{n}\right)\right\}$, by Lemma 6, we know that there exists a continuous strictly increasing function $g_{1}:[0, \infty) \rightarrow[0, \infty)$, $g_{1}(0)=0$ such that for $p \in F$

$$
\begin{aligned}
\| y_{n}- & p \|^{2} \\
\leq & \alpha_{n}\left\|G\left(x_{n}\right)-p\right\|^{2}+\left(1-\alpha_{n}\right)\left\|S_{n} G\left(x_{n}\right)-p\right\|^{2} \\
& -\alpha_{n}\left(1-\alpha_{n}\right) g_{1}\left(\left\|G\left(x_{n}\right)-S_{n} G\left(x_{n}\right)\right\|\right) \\
\leq & \alpha_{n}\left\|x_{n}-p\right\|^{2}+\left(1-\alpha_{n}\right)\left\|x_{n}-p\right\|^{2} \\
& -\alpha_{n}\left(1-\alpha_{n}\right) g_{1}\left(\left\|G\left(x_{n}\right)-S_{n} G\left(x_{n}\right)\right\|\right) \\
= & \left\|x_{n}-p\right\|^{2}-\alpha_{n}\left(1-\alpha_{n}\right) g_{1}\left(\left\|G\left(x_{n}\right)-S_{n} G\left(x_{n}\right)\right\|\right) .
\end{aligned}
$$

Since $\left\{y_{n}\right\}$ and $\left\{S_{n} G\left(y_{n}\right)\right\}$ are bounded, by Lemma 6, there exists a continuous strictly increasing function $g_{2}:[0, \infty) \rightarrow$ $[0, \infty), g_{2}(0)=0$ such that for $p \in F$

$$
\begin{aligned}
& \| x_{n+1}- p \|^{2} \\
&= \| \beta_{n}\left(f\left(x_{n}\right)-p\right)+\gamma_{n}\left(y_{n}-p\right) \\
&+\delta_{n}\left(S_{n} G\left(y_{n}\right)-p\right) \|^{2} \\
&= \|\left(\gamma_{n}+\delta_{n}\right) \\
& \times\left[\frac{\gamma_{n}}{\gamma_{n}+\delta_{n}}\left(y_{n}-p\right)\right. \\
&\left.+\frac{\delta_{n}}{\gamma_{n}+\delta_{n}}\left(S_{n} G\left(y_{n}\right)-p\right)\right] \\
&+\beta_{n}\left(f\left(x_{n}\right)-p\right) \|^{2} \\
& \leq\left(\gamma_{n}+\delta_{n}\right)^{2} \| \frac{\gamma_{n}}{\gamma_{n}+\delta_{n}}\left(y_{n}-p\right) \\
&+\frac{\delta_{n}}{\gamma_{n}+\delta_{n}}\left(S_{n} G\left(y_{n}\right)-p\right) \|^{2} \\
&+2 \beta_{n}\left\|f\left(x_{n}\right)-p\right\|\left\|x_{n+1}-p\right\| \\
&+2 \beta_{n}\left\langle f\left(x_{n}\right)-p, J\left(x_{n+1}-p\right)\right\rangle \\
& \leq\left(\gamma_{n}+\delta_{n}\right)^{2}\left[\frac{\gamma_{n}}{\gamma_{n}+\delta_{n}}\left\|y_{n}-p\right\|^{2}\right. \\
&+\frac{\delta_{n}}{\gamma_{n}+\delta_{n}}\left\|S_{n} G\left(y_{n}\right)-p\right\|^{2} \\
&\left.-\frac{\gamma_{n} \delta_{n}}{\left(\gamma_{n}+\delta_{n}\right)^{2}} g_{2}\left(\left\|y_{n}-S_{n} G\left(y_{n}\right)\right\|\right)\right] \\
& \\
&+
\end{aligned}
$$




$$
\begin{aligned}
\leq & \gamma_{n}\left\|y_{n}-p\right\|^{2}+\delta_{n}\left\|y_{n}-p\right\|^{2} \\
& -\gamma_{n} \delta_{n} g_{2}\left(\left\|y_{n}-S_{n} G\left(y_{n}\right)\right\|\right) \\
& +2 \beta_{n}\left\|f\left(x_{n}\right)-p\right\|\left\|x_{n+1}-p\right\| \\
\leq & \left\|y_{n}-p\right\|^{2}-\gamma_{n} \delta_{n} g_{2}\left(\left\|y_{n}-S_{n} G\left(y_{n}\right)\right\|\right) \\
& +2 \beta_{n}\left\|f\left(x_{n}\right)-p\right\|\left\|x_{n+1}-p\right\|,
\end{aligned}
$$

which together with (124) implies that

$$
\begin{aligned}
\| x_{n+1}- & p \|^{2} \\
\leq & \left\|x_{n}-p\right\|^{2}-\alpha_{n}\left(1-\alpha_{n}\right) g_{1}\left(\left\|G\left(x_{n}\right)-S_{n} G\left(x_{n}\right)\right\|\right) \\
& -\gamma_{n} \delta_{n} g_{2}\left(\left\|y_{n}-S_{n} G\left(y_{n}\right)\right\|\right) \\
& +2 \beta_{n}\left\|f\left(x_{n}\right)-p\right\|\left\|x_{n+1}-p\right\| .
\end{aligned}
$$

It immediately follows that

$$
\begin{aligned}
\alpha_{n}(1- & \left.\alpha_{n}\right) g_{1}\left(\left\|G\left(x_{n}\right)-S_{n} G\left(x_{n}\right)\right\|\right) \\
& +\gamma_{n} \delta_{n} g_{2}\left(\left\|y_{n}-S_{n} G\left(y_{n}\right)\right\|\right) \\
\leq & \left\|x_{n}-p\right\|^{2}-\left\|x_{n+1}-p\right\|^{2} \\
& +2 \beta_{n}\left\|f\left(x_{n}\right)-p\right\|\left\|x_{n+1}-p\right\| \\
\leq & \left(\left\|x_{n}-p\right\|+\left\|x_{n+1}-p\right\|\right)\left\|x_{n}-x_{n+1}\right\| \\
& +2 \beta_{n}\left\|f\left(x_{n}\right)-p\right\|\left\|x_{n+1}-p\right\| .
\end{aligned}
$$

According to condition (vi), we get

$\liminf _{n \rightarrow \infty} \delta_{n}=\liminf _{n \rightarrow \infty}\left(1-\beta_{n}-\gamma_{n}\right)=1-\limsup _{n \rightarrow \infty}\left(\beta_{n}+\gamma_{n}\right)>0$.

Since $\beta_{n} \rightarrow 0$ and $\left\|x_{n+1}-x_{n}\right\| \rightarrow 0$, we conclude from conditions (i) and (vi) that

$$
\begin{aligned}
& \lim _{n \rightarrow \infty} g_{1}\left(G\left(x_{n}\right)-S_{n} G\left(x_{n}\right)\right)=0, \\
& \lim _{n \rightarrow \infty} g_{2}\left(\left\|y_{n}-S_{n} G\left(y_{n}\right)\right\|\right)=0 .
\end{aligned}
$$

Utilizing the properties of $g_{1}$ and $g_{2}$, we have

$$
\begin{gathered}
\lim _{n \rightarrow \infty}\left\|G\left(x_{n}\right)-S_{n} G\left(x_{n}\right)\right\|=0, \\
\lim _{n \rightarrow \infty}\left\|y_{n}-S_{n} G\left(y_{n}\right)\right\|=0 .
\end{gathered}
$$

Note that

$$
\begin{aligned}
\| y_{n}- & x_{n} \| \\
= & \| x_{n+1}-x_{n}-\beta_{n}\left(f\left(x_{n}\right)-y_{n}\right) \\
& \quad-\delta_{n}\left(S_{n} G\left(y_{n}\right)-y_{n}\right) \| \\
\leq & \left\|x_{n+1}-x_{n}\right\|+\beta_{n}\left\|f\left(x_{n}\right)-y_{n}\right\| \\
& +\delta_{n}\left\|S_{n} G\left(y_{n}\right)-y_{n}\right\| .
\end{aligned}
$$

Thus, from (123), (130), and $\beta_{n} \rightarrow 0$, it follows that

$$
\lim _{n \rightarrow \infty}\left\|y_{n}-x_{n}\right\|=0 \text {. }
$$

On the other hand, from (130), we get

$$
\begin{aligned}
\lim _{n \rightarrow \infty}\left\|y_{n}-G\left(x_{n}\right)\right\|= & \lim _{n \rightarrow \infty}\left(1-\alpha_{n}\right) \\
& \times\left\|S_{n} G\left(x_{n}\right)-G\left(x_{n}\right)\right\|=0 .
\end{aligned}
$$

This together with (132) implies that

$$
\lim _{n \rightarrow \infty}\left\|x_{n}-G\left(x_{n}\right)\right\|=0 .
$$

By (130) and Lemma 7, we have

$$
\begin{aligned}
\left\|S G\left(x_{n}\right)-G\left(x_{n}\right)\right\| & \\
\leq & \left\|S G\left(x_{n}\right)-S_{n} G\left(x_{n}\right)\right\| \\
& +\left\|S_{n} G\left(x_{n}\right)-G\left(x_{n}\right)\right\| \longrightarrow 0 \quad \text { as } n \longrightarrow \infty .
\end{aligned}
$$

In terms of (134) and (135), we have

$$
\begin{aligned}
&\left\|x_{n}-S x_{n}\right\| \\
& \leq\left\|x_{n}-G\left(x_{n}\right)\right\|+\left\|G\left(x_{n}\right)-S G\left(x_{n}\right)\right\| \\
&+\left\|S G\left(x_{n}\right)-S x_{n}\right\| \\
& \leq 2\left\|x_{n}-G\left(x_{n}\right)\right\| \\
&+\left\|G\left(x_{n}\right)-S G\left(x_{n}\right)\right\| \longrightarrow 0 \text { as } n \longrightarrow \infty .
\end{aligned}
$$

Define a mapping $W x=(1-\theta) S x+\theta G(x)$, where $\theta \in(0,1)$ is a constant. Then by Lemma 9, we have that $\operatorname{Fix}(W)=\operatorname{Fix}(S) \cap$ $\operatorname{Fix}(G)=F$. We observe that

$$
\begin{aligned}
\| x_{n}- & W x_{n} \| \\
& =\left\|(1-\theta)\left(x_{n}-S x_{n}\right)+\theta\left(x_{n}-G\left(x_{n}\right)\right)\right\| \\
& \leq(1-\theta)\left\|x_{n}-S x_{n}\right\|+\theta\left\|x_{n}-G\left(x_{n}\right)\right\| .
\end{aligned}
$$

From (134) and (136), we obtain

$$
\lim _{n \rightarrow \infty}\left\|x_{n}-W x_{n}\right\|=0 .
$$

Now, we claim that

$$
\limsup _{n \rightarrow \infty}\left\langle f(q)-q, J\left(x_{n}-q\right)\right\rangle \leq 0,
$$

where $q=s-\lim _{t \rightarrow 0} x_{t}$ with $x_{t}$ being the fixed point of the contraction

$$
x \longmapsto t f(x)+(1-t) W x
$$

Then $x_{t}$ solves the fixed point equation $x_{t}=t f\left(x_{t}\right)+(1-$ t)Wx . Thus we have

$$
\left\|x_{t}-x_{n}\right\|=\left\|(1-t)\left(W x_{t}-x_{n}\right)+t\left(f\left(x_{t}\right)-x_{n}\right)\right\| .
$$


By Lemma 3, we conclude that

$$
\begin{aligned}
\left\|x_{t}-x_{n}\right\|^{2} & \\
\leq & (1-t)^{2}\left\|W x_{t}-x_{n}\right\|^{2} \\
& +2 t\left\langle f\left(x_{t}\right)-x_{n}, J\left(x_{t}-x_{n}\right)\right\rangle \\
\leq & (1-t)^{2}\left(\left\|W x_{t}-W x_{n}\right\|+\left\|W x_{n}-x_{n}\right\|\right)^{2} \\
& +2 t\left\langle f\left(x_{t}\right)-x_{n}, J\left(x_{t}-x_{n}\right)\right\rangle \\
\leq & (1-t)^{2}\left[\left\|x_{t}-x_{n}\right\|^{2}+2\left\|x_{t}-x_{n}\right\|\right. \\
& \left.\quad \times\left\|W x_{n}-x_{n}\right\|+\left\|W x_{n}-x_{n}\right\|^{2}\right] \\
& +2 t\left\langle f\left(x_{t}\right)-x_{t}, J\left(x_{t}-x_{n}\right)\right\rangle \\
& +2 t\left\langle x_{t}-x_{n}, J\left(x_{t}-x_{n}\right)\right\rangle \\
= & \left(1-2 t+t^{2}\right)\left\|x_{t}-x_{n}\right\|^{2}+f_{n}(t) \\
& +2 t\left\langle f\left(x_{t}\right)-x_{t}, J\left(x_{t}-x_{n}\right)\right\rangle+2 t\left\|x_{t}-x_{n}\right\|^{2},
\end{aligned}
$$

where

$$
\begin{aligned}
f_{n}(t)= & (1-t)^{2}\left(2\left\|x_{t}-x_{n}\right\|+\left\|x_{n}-W x_{n}\right\|\right) \\
& \times\left\|x_{n}-W x_{n}\right\| \longrightarrow 0 \text { as } n \longrightarrow \infty .
\end{aligned}
$$

It follows from (142) that

$$
\left\langle x_{t}-f\left(x_{t}\right), J\left(x_{t}-x_{n}\right)\right\rangle \leq \frac{t}{2}\left\|x_{t}-x_{n}\right\|^{2}+\frac{1}{2 t} f_{n}(t) \text {. }
$$

Letting $n \rightarrow \infty$ in (144) and noticing (143), we derive

$$
\limsup _{n \rightarrow \infty}\left\langle x_{t}-f\left(x_{t}\right), J\left(x_{t}-x_{n}\right)\right\rangle \leq \frac{t}{2} M_{2}
$$

where $M_{2}>0$ is a constant such that $\left\|x_{t}-x_{n}\right\|^{2} \leq M_{2}$ for all $t \in(0,1)$ and $n \geq 0$. Taking $t \rightarrow 0$ in (145), we have

$$
\limsup _{t \rightarrow 0} \limsup _{n \rightarrow \infty}\left\langle x_{t}-f\left(x_{t}\right), J\left(x_{t}-x_{n}\right)\right\rangle \leq 0 .
$$

On the other hand, we have

$$
\begin{aligned}
\langle f(q) & \left.-q, J\left(x_{n}-q\right)\right\rangle \\
= & \left\langle f(q)-q, J\left(x_{n}-q\right)-J\left(x_{n}-x_{t}\right)\right\rangle \\
& +\left\langle x_{t}-q, J\left(x_{n}-x_{t}\right)\right\rangle \\
& +\left\langle f(q)-f\left(x_{t}\right), J\left(x_{n}-x_{t}\right)\right\rangle \\
& +\left\langle f\left(x_{t}\right)-x_{t}, J\left(\mathrm{x}_{n}-x_{t}\right)\right\rangle .
\end{aligned}
$$

Hence it follows that

$$
\begin{aligned}
\limsup _{n \rightarrow \infty} & \left\langle f(q)-q, J\left(x_{n}-q\right)\right\rangle \\
\leq & \limsup _{n \rightarrow \infty}\left\langle f(q)-q, J\left(x_{n}-q\right)-J\left(x_{n}-x_{t}\right)\right\rangle \\
& +\left\|x_{t}-q\right\| \limsup _{n \rightarrow \infty}\left\|x_{n}-x_{t}\right\| \\
& +\rho\left\|q-x_{t}\right\| \limsup _{n \rightarrow \infty}\left\|x_{n}-x_{t}\right\| \\
& +\limsup _{n \rightarrow \infty}\left\langle f\left(x_{t}\right)-x_{t}, J\left(x_{n}-x_{t}\right)\right\rangle .
\end{aligned}
$$

Taking into account that $x_{t} \rightarrow q$ as $t \rightarrow 0$, we have from (146)

$$
\begin{aligned}
\limsup _{n \rightarrow \infty} & \left\langle f(q)-q, J\left(x_{n}-q\right)\right\rangle \\
\quad & =\limsup _{t \rightarrow 0} \limsup _{n \rightarrow \infty}\left\langle f(q)-q, J\left(x_{n}-q\right)\right\rangle \\
\quad \leq & \limsup _{t \rightarrow 0} \limsup _{n \rightarrow \infty}\left\langle f(q)-q, J\left(x_{n}-q\right)-J\left(x_{n}-x_{t}\right)\right\rangle .
\end{aligned}
$$

Since $X$ has a uniformly Gâteaux differentiable norm, the duality mapping $J$ is norm-to-weak ${ }^{*}$ uniformly continuous on bounded subsets of $X$. Consequently, the two limits are interchangeable, and hence (139) holds. From (123), we get $\left(x_{n+1}-q\right)-\left(x_{n}-q\right) \rightarrow 0$. Noticing the norm-to-weak ${ }^{*}$ uniform continuity of $J$ on bounded subsets of $X$, we deduce from (139) that

$$
\begin{aligned}
& \limsup _{n \rightarrow \infty}\left\langle f(q)-q, J\left(x_{n+1}-q\right)\right\rangle \\
&=\limsup _{n \rightarrow \infty}\left(\left\langle f(q)-q, J\left(x_{n+1}-q\right)-J\left(x_{n}-q\right)\right\rangle\right. \\
&\left.\quad+\left\langle f(q)-q, J\left(x_{n+1}-q\right)\right\rangle\right) \\
&=\limsup _{n \rightarrow \infty}\left\langle f(q)-q, J\left(x_{n}-q\right)\right\rangle \leq 0 .
\end{aligned}
$$

Finally, let us show that $x_{n} \rightarrow q$ as $n \rightarrow \infty$. We observe that

$$
\begin{aligned}
\left\|y_{n}-q\right\|= & \| \alpha_{n}\left(G\left(x_{n}\right)-q\right) \\
& \quad+\left(1-\alpha_{n}\right)\left(S_{n} G\left(x_{n}\right)-q\right) \| \\
\leq & \alpha_{n}\left\|x_{n}-q\right\|+\left(1-\alpha_{n}\right)\left\|x_{n}-q\right\| \\
= & \left\|x_{n}-q\right\|,
\end{aligned}
$$




$$
\begin{aligned}
\left\|x_{n+1}-q\right\|^{2}= & \beta_{n}\left\langle f\left(x_{n}\right)-f(q)+f(q)-q, J\left(x_{n+1}-q\right)\right\rangle \\
& +\left\langle\gamma_{n}\left(y_{n}-q\right)\right. \\
& \left.+\delta_{n}\left(S_{n} G\left(y_{n}\right)-q\right), J\left(x_{n+1}-q\right)\right\rangle \\
\leq & \beta_{n}\left\|f\left(x_{n}\right)-f(q)\right\|\left\|x_{n+1}-q\right\| \\
& +\beta_{n}\left\langle f(q)-q, J\left(x_{n+1}-q\right)\right\rangle \\
& +\left\|\gamma_{n}\left(y_{n}-q\right)+\delta_{n}\left(S_{n} G\left(y_{n}\right)-q\right)\right\|\left\|x_{n+1}-q\right\| \\
\leq & \beta_{n} \rho\left\|x_{n}-q\right\|\left\|x_{n+1}-q\right\| \\
& +\beta_{n}\left\langle f(q)-q, J\left(x_{n+1}-q\right)\right\rangle \\
& +\left(\gamma_{n}\left\|y_{n}-q\right\|+\delta_{n}\left\|y_{n}-q\right\|\right)\left\|x_{n+1}-q\right\| \\
= & \beta_{n} \rho\left\|x_{n}-q\right\|\left\|x_{n+1}-q\right\| \\
& +\beta_{n}\left\langle f(q)-q, J\left(x_{n+1}-q\right)\right\rangle \\
& +\left(\gamma_{n}+\delta_{n}\right)\left\|y_{n}-q\right\|\left\|x_{n+1}-q\right\| \\
\leq & \beta_{n} \rho\left\|x_{n}-q\right\|\left\|x_{n+1}-q\right\| \\
& +\beta_{n}\left\langle f(q)-q, J\left(x_{n+1}-q\right)\right\rangle \\
& +\left(1-\beta_{n}\right)\left\|x_{n}-q\right\|\left\|x_{n+1}-q\right\| \\
= & \left(1-\beta_{n}(1-\rho)\right)\left\|x_{n}-q\right\|\left\|x_{n+1}-q\right\| \\
& +\beta_{n}\left\langle f(q)-q, J\left(x_{n+1}-q\right)\right\rangle \\
\leq & \frac{1-\beta_{n}(1-\rho)}{2}\left(\left\|x_{n}-q\right\|^{2}+\left\|x_{n+1}-q\right\|^{2}\right) \\
& +\beta_{n}\left\langle f(q)-q, J\left(x_{n+1}-q\right)\right\rangle \\
\leq & \frac{1-\beta_{n}(1-\rho)}{2}\left\|x_{n}-q\right\|^{2}+\frac{1}{2}\left\|x_{n+1}-q\right\|^{2} \\
& +\beta_{n}\left\langle f(q)-q, J\left(x_{n+1}-q\right)\right\rangle . \\
& \\
& \\
&
\end{aligned}
$$

So, we have

$$
\begin{aligned}
&\left\|x_{n+1}-q\right\|^{2} \\
& \leq\left(1-\beta_{n}(1-\rho)\right)\left\|x_{n}-q\right\|^{2} \\
&+2 \beta_{n}\left\langle f(q)-q, J\left(x_{n+1}-q\right)\right\rangle \\
&=\left(1-\beta_{n}(1-\rho)\right)\left\|x_{n}-q\right\|^{2} \\
&+\beta_{n}(1-\rho) \frac{2\left\langle f(q)-q, J\left(x_{n+1}-q\right)\right\rangle}{1-\rho} .
\end{aligned}
$$

Since $\sum_{n=0}^{\infty} \beta_{n}=\infty$ and $\lim \sup _{n \rightarrow \infty}\left\langle f(q)-q, J\left(x_{n+1}-q\right)\right\rangle \leq$ 0 , by Lemma 2, we conclude from (153) that $x_{n} \rightarrow q$ as $n \rightarrow$ $\infty$. This completes the proof.

Corollary 23. Let $C$ be a nonempty, closed, and convex subset of a uniformly convex Banach space $X$ which has a uniformly
Gâteaux differentiable norm. Let $\Pi_{C}$ be a sunny nonexpansive retraction from $X$ onto $C$. Let the mapping $B_{i}: C \rightarrow X$ be $\lambda_{i^{-}}$ strictly pseudocontractive and $\alpha_{i}$-strongly accretive with $\alpha_{i}+$ $\lambda_{i} \geq 1$ for $i=1$, 2. Let $f: C \rightarrow C$ be a contraction with coefficient $\rho \in(0,1)$. Let $S$ be a nonexpansive mapping of $C$ into itself such that $F=\operatorname{Fix}(S) \cap \Omega \neq \emptyset$, where $\Omega$ is the fixed point set of the mapping $G=\Pi_{C}\left(I-\mu_{1} B_{1}\right) \Pi_{C}\left(I-\mu_{2} B_{2}\right)$. For arbitrarily given $x_{0} \in C$, let $\left\{x_{n}\right\}$ be the sequence generated by

$$
\begin{gathered}
y_{n}=\alpha_{n} G\left(x_{n}\right)+\left(1-\alpha_{n}\right) S G\left(x_{n}\right), \\
x_{n+1}=\beta_{n} f\left(x_{n}\right)+\gamma_{n} y_{n}+\delta_{n} S G\left(y_{n}\right), \quad \forall n \geq 0,
\end{gathered}
$$

where $1-\left(\lambda_{i} /\left(1+\lambda_{i}\right)\right)\left(1-\sqrt{\left(1-\alpha_{i}\right) / \lambda_{i}}\right) \leq \mu_{i} \leq 1$ for $i=1,2$. Suppose that $\left\{\alpha_{n}\right\},\left\{\beta_{n}\right\},\left\{\gamma_{n}\right\}$, and $\left\{\delta_{n}\right\}$ are the sequences in $(0,1)$ satisfying the following conditions:

(i) $0<\liminf _{n \rightarrow \infty} \alpha_{n} \leq \lim \sup _{n \rightarrow \infty} \alpha_{n}<1$,

(ii) $\lim _{n \rightarrow \infty} \beta_{n}=0$ and $\sum_{n=0}^{\infty} \beta_{n}=\infty$,

(iii) $\sum_{n=1}^{\infty}\left|\alpha_{n}-\alpha_{n-1}\right|<\infty$ or $\lim _{n \rightarrow \infty}\left|\alpha_{n}-\alpha_{n-1}\right| / \beta_{n}=0$,

(iv) $\sum_{n=1}^{\infty}\left|\beta_{n}-\beta_{n-1}\right|<\infty$ or $\lim _{n \rightarrow \infty} \beta_{n-1} / \beta_{n}=1$,

(v) $\sum_{n=1}^{\infty}\left|\left(\gamma_{n} /\left(1-\beta_{n}\right)\right)-\left(\gamma_{n-1} /\left(1-\beta_{n-1}\right)\right)\right|<\infty$ or $\lim _{n \rightarrow \infty}\left(1 / \beta_{n}\right)\left|\left(\gamma_{n} /\left(1-\beta_{n}\right)\right)-\left(\gamma_{n-1} /\left(1-\beta_{n-1}\right)\right)\right|=0$,

(vi) $0<\liminf _{n \rightarrow \infty} \gamma_{n} \leq \lim \sup _{n \rightarrow \infty} \gamma_{n}<1$.

Then $\left\{x_{n}\right\}$ converges strongly to $q \in F$, which solves the following VIP:

$$
\langle q-f(q), J(q-p)\rangle \leq 0, \quad \forall p \in F .
$$

Further, we illustrate Theorem 22 by virtue of an example, that is, the following corollary.

Corollary 24. Let $C$ be a nonempty, closed, and convex subset of a uniformly convex Banach space $X$ which has a uniformly Gâteaux differentiable norm. Let $\Pi_{C}$ be a sunny nonexpansive retraction from $X$ onto $C$. Let $f: C \rightarrow C$ be a contraction with coefficient $\rho \in(0,1)$. Let $T: C \rightarrow C$ be a self-mapping on $C$ such that $I-T$ is $\zeta$-strictly pseudocontractive and $\theta$-strongly accretive with $\zeta+\theta \geq 1$, and let $S$ be a nonexpansive mapping of C into itself such that $F=\operatorname{Fix}(S) \cap \operatorname{Fix}(T) \neq \emptyset$. For arbitrarily given $x_{0} \in C$, let $\left\{x_{n}\right\}$ be the sequence generated by

$$
\begin{gathered}
y_{n}=\alpha_{n} G\left(x_{n}\right)+\left(1-\alpha_{n}\right) S(I-\lambda(I-T)) x_{n}, \\
x_{n+1}=\beta_{n} f\left(x_{n}\right)+\gamma_{n} y_{n}+\delta_{n} S(I-\lambda(I-T)) y_{n}, \quad \forall n \geq 0,
\end{gathered}
$$

where $1-(\zeta /(1+\zeta))(1-\sqrt{(1-\theta) / \zeta}) \leq \lambda \leq 1$. Suppose that $\left\{\alpha_{n}\right\},\left\{\beta_{n}\right\},\left\{\gamma_{n}\right\}$, and $\left\{\delta_{n}\right\}$ are the sequences in $(0,1)$ satisfying the following conditions:

(i) $0<\liminf _{n \rightarrow \infty} \alpha_{n} \leq \lim \sup _{n \rightarrow \infty} \alpha_{n}<1$,

(ii) $\lim _{n \rightarrow \infty} \beta_{n}=0$ and $\sum_{n=0}^{\infty} \beta_{n}=\infty$,

(iii) $\sum_{n=1}^{\infty}\left|\alpha_{n}-\alpha_{n-1}\right|<\infty$ or $\lim _{n \rightarrow \infty}\left|\alpha_{n}-\alpha_{n-1}\right| / \beta_{n}=0$,

(iv) $\sum_{n=1}^{\infty}\left|\beta_{n}-\beta_{n-1}\right|<\infty$ or $\lim _{n \rightarrow \infty} \beta_{n-1} / \beta_{n}=1$, 
(v) $\sum_{n=1}^{\infty}\left|\left(\gamma_{n} /\left(1-\beta_{n}\right)\right)-\left(\gamma_{n-1} /\left(1-\beta_{n-1}\right)\right)\right|<\infty$ or $\lim _{n \rightarrow \infty}\left(1 / \beta_{n}\right)\left|\left(\gamma_{n} /\left(1-\beta_{n}\right)\right)-\left(\gamma_{n-1} /\left(1-\beta_{n-1}\right)\right)\right|=0$,

(vi) $0<\liminf _{n \rightarrow \infty} \gamma_{n} \leq \limsup _{n \rightarrow \infty} \gamma_{n}<1$.

Then $\left\{x_{n}\right\}$ converges strongly to $q \in F$, which solves the following VIP:

$$
\langle q-f(q), J(q-p)\rangle \leq 0, \quad \forall p \in F .
$$

Proof. Utilizing the arguments similar to those in the proof of Corollary 16, we can obtain the desired result.

Remark 25. As previous, we emphasize that our composite iterative algorithms (i.e., the iterative schemes (27) and (111)) are based on Korpelevich's extragradient method and viscosity approximation method. It is well known that the so-called viscosity approximation method must contain a contraction $f$ on $C$. In the meantime, it is worth pointing out that our proof of Theorems 14 and 22 must make use of Lemma 8 for implicit viscosity approximation method; that is, Lemma 8 plays a key role in our proof of Theorems 14 and 22. Therefore, there is no doubt that the contraction $f$ in Theorems 14 and 22 cannot be replaced by a general $k$ Lipschitzian mapping with constant $k \geq 0$.

Remark 26. Theorem 22 improves, extends, supplements, and develops [2, Theorem 3.1 and Corollary 3.2] and [5, Theorems 3.1] in the following aspects.

(i) The problem of finding a point $q \in \bigcap_{n} \operatorname{Fix}\left(S_{n}\right) \cap \Omega$ in Theorem 22 is more general and more subtle than the problem of finding a point $q \in \operatorname{Fix}(S) \cap \operatorname{VI}(C, A)$ in Jung [5, Theorem 3.1].

(ii) The iterative scheme in [2, Theorem 3.1] is extended to develop the iterative scheme (111) of Theorem 22 by virtue of the iterative scheme of [5, Theorem 3.1]. The iterative scheme (111) in Theorem 22 is more advantageous and more flexible than the iterative scheme in [2, Theorem 3.1] because it involves several parameter sequences $\left\{\alpha_{n}\right\},\left\{\beta_{n}\right\},\left\{\gamma_{n}\right\}$, and $\left\{\delta_{n}\right\}$.

(iii) The iterative scheme (111) in Theorem 22 is very different from everyone in both [2, Theorem 3.1] and [5, Theorem 3.1] because the mappings $S_{n}$ and $G$ in the iterative scheme of [2, Theorem 3.1] and the mapping $S P_{C}\left(I-\lambda_{n} A\right)$ in the iterative scheme of [5, Theorem 3.1] are replaced by the same composite mapping $S_{n} G$ in the iterative scheme (111) of Theorem 22.

(iv) The proof in [2, Theorem 3.1] depends on the argument techniques in [3], the inequality in 2-uniformly smooth Banach spaces, and the inequality in smooth and uniform convex Banach spaces. However, the proof of Theorem 22 does not depend on the argument techniques in [3], the inequality in 2-uniformly smooth Banach spaces, and the inequality in smooth and uniform convex Banach spaces. It depends on only the inequality in uniform convex Banach spaces.

(v) The assumption of the uniformly convex and 2uniformly smooth Banach space $X$ in [2, Theorem 3.1] is weakened to the one of the uniformly convex Banach space $X$ having a uniformly Gateaux differentiable norm in Theorem 22.

(vi) The iterative scheme in [2, Corollary 3.2] is extended to develop the new iterative scheme in Corollary 15 because the mappings $S$ and $G$ are replaced by the same composite mapping SG in Corollary 23.

Finally, we observe that related results can be found in recent papers, for example, [15-24] and the references therein.

\section{Acknowledgments}

This research was partially supported by the National Science Foundation of China (11071169), Innovation Program of Shanghai Municipal Education Commission (09ZZ133), and Ph.D. Program Foundation of Ministry of Education of China (20123127110002). This research was partially supported by a Grant from NSC 101-2115-M-037-001.

\section{References}

[1] Y. Takahashi, K. Hashimoto, and M. Kato, "On sharp uniform convexity, smoothness, and strong type, cotype inequalities," Journal of Nonlinear and Convex Analysis, vol. 3, no. 2, pp. 267281, 2002.

[2] G. Cai and S. Q. Bu, "Convergence analysis for variational inequality problems and fixed point problems in 2-uniformly smooth and uniformly convex Banach spaces," Mathematical and Computer Modelling, vol. 55, no. 3-4, pp. 538-546, 2012.

[3] L. C. Ceng, C. Y. Wang, and J. C. Yao, "Strong convergence theorems by a relaxed extragradient method for a general system of variational inequalities," Mathematical Methods of Operations Research, vol. 67, no. 3, pp. 375-390, 2008.

[4] G. M. Korpelevič, "An extragradient method for finding saddle points and for other problems," Ékonomika i Matematicheskie Metody, vol. 12, no. 4, pp. 747-756, 1976.

[5] J. S. Jung, "A new iteration method for nonexpansive mappings and monotone mappings in Hilbert spaces," Journal of Inequalities and Applications, vol. 2010, Article ID 251761, 16 pages, 2010.

[6] H. K. Xu, "Iterative algorithms for nonlinear operators," Journal of the London Mathematical Society 2, vol. 66, no. 1, pp. 240-256, 2002.

[7] T. Suzuki, "Strong convergence of Krasnoselskii and Mann's type sequences for one-parameter nonexpansive semigroups without Bochner integrals," Journal of Mathematical Analysis and Applications, vol. 305, no. 1, pp. 227-239, 2005.

[8] S. Reich, "Weak convergence theorems for nonexpansive mappings in Banach spaces," Journal of Mathematical Analysis and Applications, vol. 67, no. 2, pp. 274-276, 1979.

[9] H. K. Xu, "Inequalities in Banach spaces with applications," Nonlinear Analysis: Theory, Methods \& Applications, vol. 16, no. 12, pp. 1127-1138, 1991.

[10] K. Aoyama, Y. Kimura, W. Takahashi, and M. Toyoda, "Approximation of common fixed points of a countable family of nonexpansive mappings in a Banach space," Nonlinear Analysis: Theory, Methods \& Applications, vol. 67, no. 8, pp. 2350-2360, 2007.

[11] J. S. Jung, "Iterative approaches to common fixed points of nonexpansive mappings in Banach spaces," Journal of Mathematical Analysis and Applications, vol. 302, no. 2, pp. 509-520, 2005. 
[12] H. K. Xu, "Viscosity approximation methods for nonexpansive mappings," Journal of Mathematical Analysis and Applications, vol. 298, no. 1, pp. 279-291, 2004.

[13] R. E. Bruck, "Properties of fixed-point sets of nonexpansive mappings in Banach spaces," Transactions of the American Mathematical Society, vol. 179, pp. 251-262, 1973.

[14] S. Kamimura and W. Takahashi, "Strong convergence of a proximal-type algorithm in a Banach space," SIAM Journal on Optimization, vol. 13, no. 3, pp. 938-945, 2002.

[15] L. C. Ceng and J. C. Yao, "An extragradient-like approximation method for variational inequality problems and fixed point problems," Applied Mathematics and Computation, vol. 190, no. 1, pp. 205-215, 2007.

[16] L. C. Zeng and J. C. Yao, "Strong convergence theorem by an extragradient method for fixed point problems and variational inequality problems," Taiwanese Journal of Mathematics, vol. 10, no. 5, pp. 1293-1303, 2006.

[17] L. C. Ceng, Q. H. Ansari, and J. C. Yao, "An extragradient method for solving split feasibility and fixed point problems," Computers \& Mathematics with Applications, vol. 64, no. 4, pp. 633-642, 2012.

[18] L. C. Ceng, M. Teboulle, and J. C. Yao, "Weak convergence of an iterative method for pseudomonotone variational inequalities and fixed-point problems," Journal of Optimization Theory and Applications, vol. 146, no. 1, pp. 19-31, 2010.

[19] L. C. Ceng, Q. H. Ansari, and J. C. Yao, "Relaxed extragradient methods for finding minimum-norm solutions of the split feasibility problem," Nonlinear Analysis: Theory, Methods \& Applications, vol. 75, no. 4, pp. 2116-2125, 2012.

[20] L. C. Ceng, Q. H. Ansari, and J. C. Yao, "Relaxed extragradient iterative methods for variational inequalities," Applied Mathematics and Computation, vol. 218, no. 3, pp. 1112-1123, 2011.

[21] L. C. Ceng, Q. H. Ansari, N. C. Wong, and J. C. Yao, "An extragradient-like approximation method for variational inequalities and fixed point problems," Fixed Point Theory and Applications, vol. 2011, article 22, 18 pages, 2011.

[22] L. C. Ceng, S. M. Guu, and J. C. Yao, "Finding common solutions of a variational inequality, a general system of variational inequalities, and a fixed-point problem via a hybrid extragradient method," Fixed Point Theory and Applications, vol. 2011, Article ID 626159, 22 pages, 2011.

[23] Y. Yao and J. C. Yao, "On modified iterative method for nonexpansive mappings and monotone mappings," Applied Mathematics and Computation, vol. 186, no. 2, pp. 1551-1558, 2007.

[24] L. C. Ceng, H. K. Xu, and J. C. Yao, "Strong convergence of an iterative method with perturbed mappings for nonexpansive and accretive operators," Numerical Functional Analysis and Optimization, vol. 29, no. 3-4, pp. 324-345, 2008. 


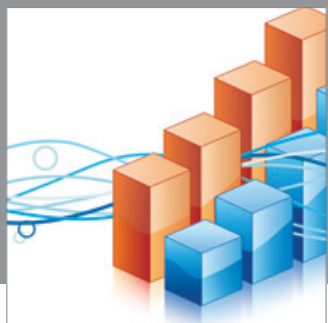

Advances in

Operations Research

mansans

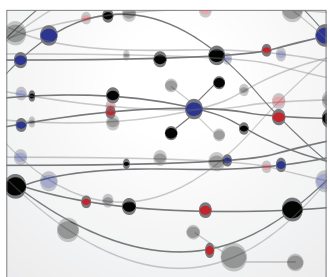

The Scientific World Journal
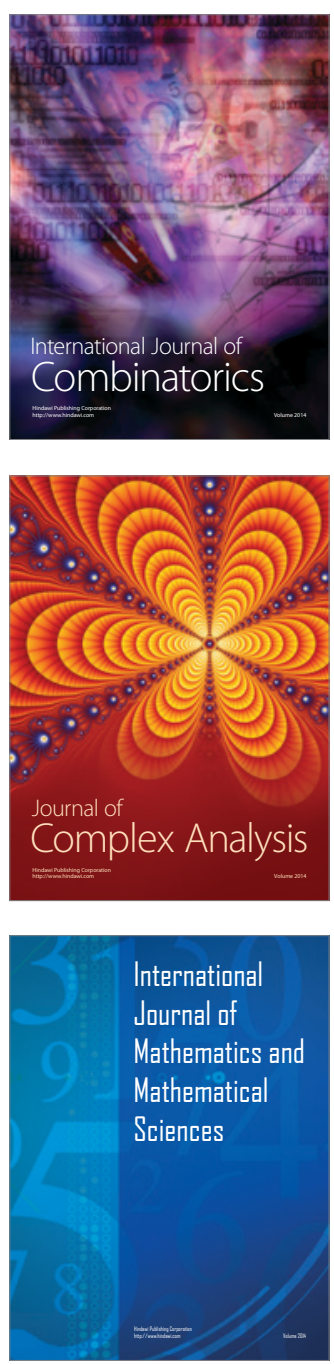
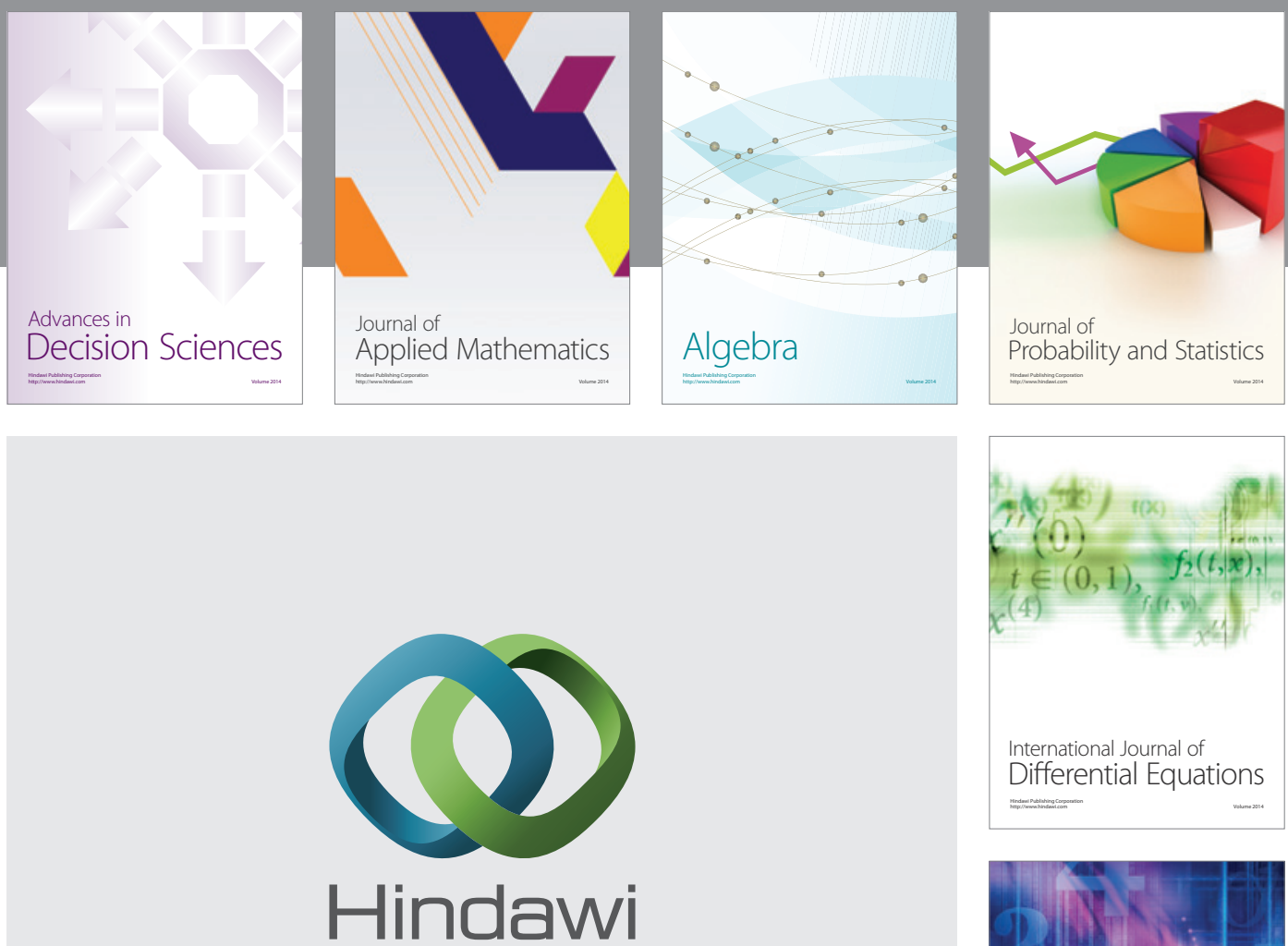

Submit your manuscripts at http://www.hindawi.com
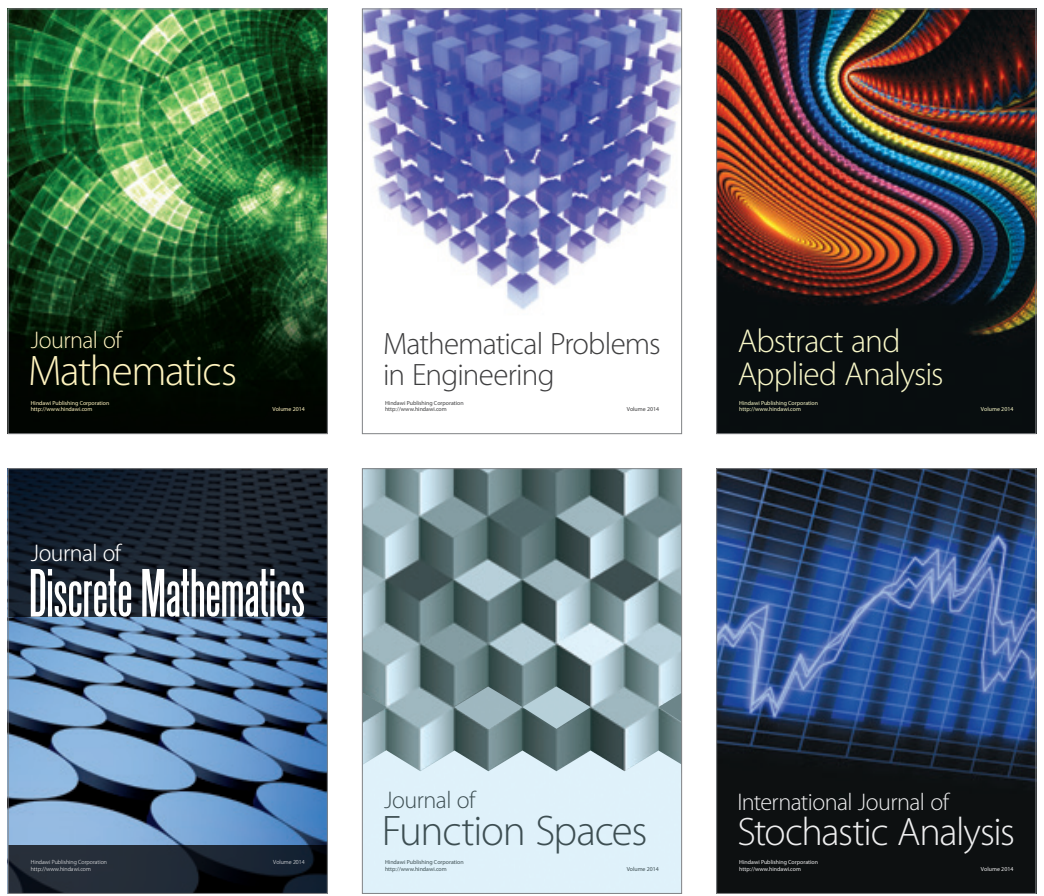

Journal of

Function Spaces

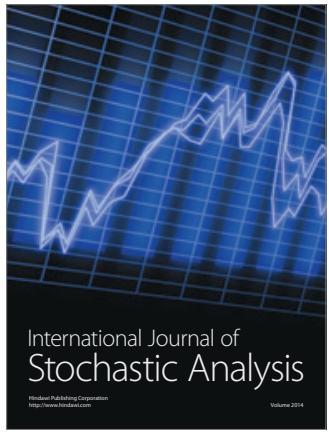

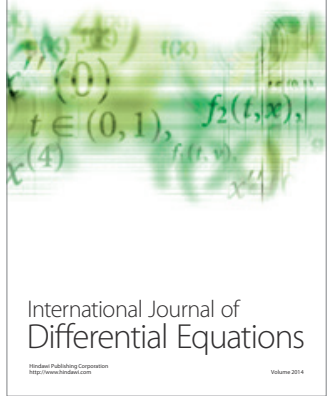
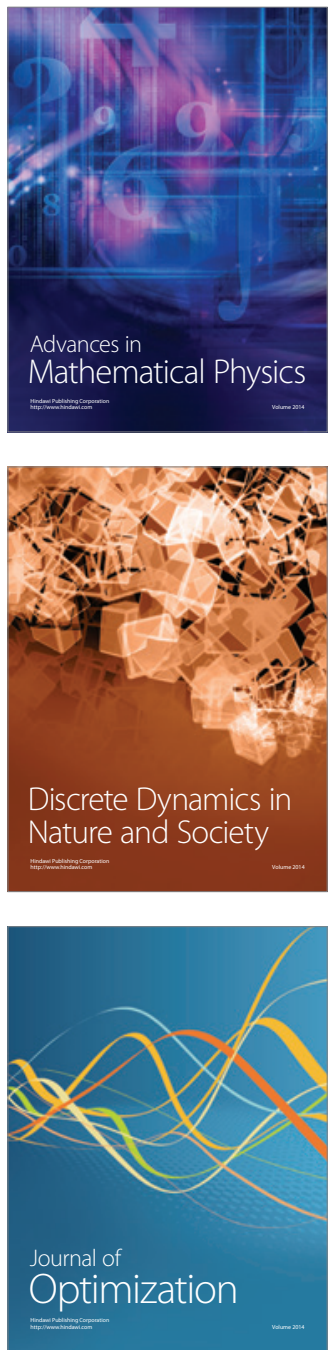